Estabilidade do Filtro de Kalman para Sistemas Lineares com Saltos Markovianos Maria Josiane Ferreira Gomes 
SERVIÇO DE PÓS-GRADUAÇÃO DO ICMC-USP

Data de Depósito: 02/03/2010

Assinatura:

\title{
Estabilidade do Filtro de Kalman para Sistemas Lineares com Saltos Markovianos
}

\author{
Maria Josiane Ferreira Gomes \\ Orientador: Prof. Dr. Eduardo Fontoura Costa
}

Dissertação apresentada ao Instituto de Ciências Matemáticas e de Computação - ICMC-USP, como parte dos requisitos para obtenção do título de Mestre em Ciências - Ciências de Computação e Matemática Computacional.

USP - São Carlos

Março/2010 
"A fé não consiste em saber qual é o mistério do universo, mas sim em ter a conviç̧ão de que existe um mistério e ele é maior do que nós".

Rabino David Wolpe 



\section{Agradecimentos}

Tentarei expressar em poucas linhas minha gratidão às pessoas que de alguma forma estiveram presentes e que muito me ajudaram em meu caminho até aqui.

À Deus, pela constante e amorosa presença.

À minha família, que sempre me deu suporte de forma incondicional em todos os aspectos possíveis durante meus estudos. Aos meus pais, Vicente e Jovina, pela dedicação, pela educação e pelo esforço para comigo e meus irmãos. Agradeço-os ainda pelo exemplo de pessoas íntegras que são. Aos meus irmãos, Carlos, Claudinei, Márcio, Gil, Jonas e Alexandre, e às minhas irmãs, Roseli e Eliane, a quem devo tudo que sou.

Ao Prof. Dr. Eduardo Fontoura Costa, pela amizade, competência, seriedade e pela forma generosa com que compartilhou seus conhecimentos nestes dois anos de trabalho.

Aos professores Ivo Machado da Costa e José Ruidival dos Santos Filho, pelos valorosos ensinamentos no curso de graduação.

À CAPES - Coordenação de Aperfeiçoamento de Pessoal de Nível Superior, pelo suporte financeiro.

À todos os professores e funcionários do Instituto de Ciências Matemáticas e de Computação da Universidade de São Paulo.

À todos os amigos e colegas, em especial, a Aline, Giseli, Laís e Letrícia.

Meus sinceros agradecimentos. 

O filtro de Kalman é amplamente conhecido e utilizado em aplicações, em virtude de apresentar diversas propriedades interessantes. Este trabalho aborda uma das características mais importantes, a estabilidade do filtro de Kalman aplicado a sistemas lineares discretos com saltos Markovianos. Sistemas desta classe são muito empregados em problemas práticos.

Neste trabalho mostramos que o conceito de controlabilidade fraca e detetabilidade estocástica são condições suficientes para estabilidade do filtro de Kalman com relação a condição inicial. No que se refere a estabilidade no sentido mais usual, apresentamos resultados parciais, dependentes de uma condição adicional sobre a cadeia de Markov, bem como uma conjectura.

O estudo da estabilidade do filtro de Kalman é relevante, pois filtros instáveis oferecem estimativas de baixa qualidade. O tema tem interesse teórico inerente e é bastante relevante para aplicações. 

Kalman filters present several interesting features that make them relevant for many applications. In this work we study one of the main issues in Kalman filtering - stability. We deal with Kalman filters for Markov jump linear systems, a class of systems with applications in many different areas.

We consider the concepts of weak controllability and stochastic detectability and we show that they ensure stability of the Kalman filter with respect to the initial condition. As for the stability, we present some results relying in a conjecture and an additional condition on the Markov chain.

The study of the stability of the Kalman filter is important, since unstable filters may lead to poor estimates. The stability issue has inherent theoretical interest and is relevant for applications. 

1 Introdução $\quad 15$

1.1 Notações . . . . . . . . . . . . . . . . . . . . . . . . . . . 17

1.2 Organização do texto . . . . . . . . . . . . . . . . 18

2 Conceitos e Resultados Preliminares $\quad 19$

2.1 Sistemas Lineares . . . . . . . . . . . . . . . . . . . . . . . . . . 19

2.1.1 Propriedades usuais . . . . . . . . . . . . . . 21

2.2 Cadeia de Markov . . . . . . . . . . . . . . . . . . . . . . . 21

2.3 Os SLSMs . . . . . . . . . . . . . . . . . . . . . . . . . . 23

2.4 Filtro de Kalman para Sistemas Lineares com Saltos Markovianos . . . 24

3 W-controlabilidade $\quad 29$

3.1 Controlabilidade para SLSM . . . . . . . . . . . . . . . . . 29

3.2 Exemplos Numéricos . . . . . . . . . . . . . . . . . . . 32

3.3 Positividade da covariância . . . . . . . . . . . . . . . 33

4 Estabilidade do FK com relação a condição inicial $\quad 37$

4.1 Estabilidade do FK em relação a $\Psi$. . . . . . . . . . . . . . . . . . . 37

4.2 Exemplos Numéricos . . . . . . . . . . . . . . . . . 40

5 Estabilidade do Filtro de Kalman $\quad 45$

5.1 Abordagem usual de estabilidade do FK . . . . . . . . . . . . . 45

5.2 Exemplos Numéricos ...................... 52

6 Conclusões $\quad 57$

A Teste de w-controlabilidade $\quad 59$ 

Lista de Tabelas

4.1 Tabela resumo: Estabilidade c.r $\Psi \ldots \ldots$. . . . . . . . . . . 43

5.1 Tabela resumo: Estabilidade do FK . . . . . . . . . . . . . 56 



\section{Lista de Figuras}

2.1 Representação da cadeia de Markov com três estados. . . . . . . . . . . 22

2.2 Modelo físico de um Sistema de Suspensão com Saltos Markovianos . . 24

4.1 Curvas para o Exemplo 4.1: Covariância do erro de estimação . . . . 38

4.2 Exemplo 4.2: w-controlável e s-detetável. Estimativas para $P(k)$ e $\mathcal{E}\{W(k, \Psi)\}$ e os respectivos desvios padrões marcados com linha contínua. . . . . . 41

4.3 Exemplo 4.3: Não w-controlável e s-detetável. Estimativas para $P(k)$ e $\mathcal{E}\{W(k, \Psi)\}$ e os respectivos desvios padrões marcados com linha contínua. 42

4.4 Exemplo 4.4: Não w-controlável e s-detetável. Estimativas para $P(k)$ e $\mathcal{E}\{W(k, \Psi)\}$ e os respectivos desvios padrões marcados com linha contínua. 43

4.5 Exemplo 4.5: w-controlável e não s-detetável. Estimativas para $P(k)$ e $\mathcal{E}\{W(k, \Psi)\}$ e os respectivos desvios padrões marcados com linha contínua. 44

5.1 Exemplo 5.1: w-controlável e s-detetável. Estimativas para $P(k)$ e $\mathcal{E}\{W(k, B, \Psi)\}$ e os respectivos desvios padrões marcados com linha contínua. . . . . . 53

5.2 Exemplo 5.2: não w-controlável e s-detetável. Estimativas para $P(k)$ e $\mathcal{E}\{W(k, B, \Psi)\}$ e os respectivos desvios padrões marcados com linha contínua. . . . . . . . . . . . . . . . .

5.3 Exemplo 5.3: não w-controlável e s-detetável. Estimativas para $P(k)$ e $\mathcal{E}\{W(k, B, \Psi)\}$ e os respectivos desvios padrões marcados com linha contínua. . . . . . . . . . . . . . . . .

5.4 Exemplo 5.4: w-controlável e não s-detetável. Estimativas para $P(k)$ e $\mathcal{E}\{W(k, B, \Psi)\}$ e os respectivos desvios padrões marcados com linha continua. .......................... 56 



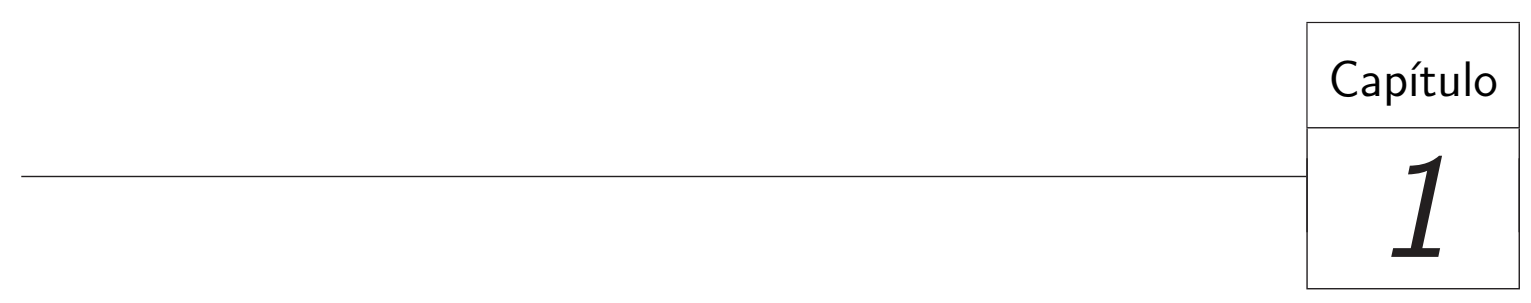

Introdução

Neste capítulo, fazemos a apresentação do cenário no qual desenvolvemos o trabalho. Apresentamos também, nas seções seguintes, algumas notações adotadas no desenvolvimento do trabalho e a organização do texto.

O Filtro de Kalman (FK) é conhecido desde a década de sessenta, quando foi introduzido por Rudolph Kalman, no desenvolvimento de um processo recursivo para solucionar problemas lineares ligados à filtragem a tempo discreto. O FK é um processo recursivo de estimação muito eficiente, tem como base a estimação da variável de estado através da variável observada, de forma a estimar os estados passados, atuais e futuros (respectivamente, fala-se em suavização, filtragem e predição). As aplicações do FK são vastas, são inúmeros os trabalhos publicados fazendo uso do filtro de Kalman, para um estudo interessante referenciamos [26], sendo clássicas as referências [1] e [46].

Neste trabalho, o FK é abordado para sistemas lineares com saltos Markovianos (SLSM), que como veremos adiante, são sistemas lineares que apresentam mudanças abruptas de comportamento. Assim como nas pesquisas referidas adiante, adotamos dois modelos para análise. O primeiro sendo o modelo atual, também conhecido como modelo real, no qual denotamos as matrizes de ruído aditivo de estado (que formalizamos mais tarde) e covariância inicial respectivamente por $B$ e $\Psi$. O segundo é o modelo nominal, no qual consideramos as informações disponíveis, com as quais realizamos o cálculo do ganho do filtro de Kalman. Denotamos as matrizes de ruído e covariância inicial respectivamente por $E$ e $\Sigma$. 
O FK possui propriedades muito vantajosas, é ótimo em vários sentidos, apresenta linearidade e os ganhos são pré-calculados. Para maiores detalhes veja [1]. Por outro lado, é comum em situações práticas, na obtenção de modelos, o surgimento de erros em alguns termos do sistema, que podem causar a perda de qualidade de estimação, tornando o filtro de Kalman menos eficiente. Em alguns casos esta perda de qualidade leva a divergência da covariância do erro de estimação real, surgindo a necessidade do estudo de condições que sejam suficientes ou necessárias para que exista limitação da covariância do erro de estimação real, ou seja, a estabilidade do FK. A estabilidade do FK tem sido estudada no contexto de sistemas lineares discretos (SLD) invariantes no tempo. Dentre algumas referências importantes, citamos [6], [18], [21], [25], [37], [38], [40] e [45]. É um fato conhecido que o FK sob a condição de um modelo com informações incorretas pode ser divergente, de maneira que o erro de estimação pode apresentar uma rápida divergência do valor verdadeiro, veja [37]. Esta questão tem sido estudada em diferentes abordagens e para SLDs invariantes no tempo, condições necessárias e suficientes para estabilidade do FK foram exploradas para o filtro recursivo, veja [6] e [7]. A bibligrafia apresenta resultados sobre a divergência do FK, os quais são considerados em cenários simples, impondo-se detetabilidade como condição para a existência de limitantes estacionários ou soluções periódicas para a matriz de Riccati, veja [37] e [40].

No contexto em questão, a partir de informações do modelo nominal, buscamos obter condições de estabilidade para o FK aplicado ao modelo real. O problema é formulado a seguir. Assuma que o FK seja calculado para os valores nominais, o qual é caracterizado por certas matrizes de covariância $E_{i}, \quad i=1,2, \ldots, N$ e $\Sigma$. Utilizamos os valores do filtro calculado a partir dos valores nominais para obter a covariância do erro de estimação real, denotada por $W(k)$, caracterizada pelas matrizes de covariância do erro real $B_{i}$ e $\Psi$ respectivamente. Em geral, temos $\Sigma \neq \Psi$ e $E_{i} \neq B i$, detalhamos cada passagem desta estapa na Seção 2.4.

Assuma que a covariância do erro de estimação nominal no instante de tempo $k$, aqui denotada por $P(k), k \geq 0$ tenha valor esperado limitado, $\mathcal{E}\{P(k)\} \leq \bar{P}$. É calculado a covariância do erro de estimação real $W(k), k \geq 0$ e analisada as condições suficientes para obter limitação, que é equivalente a estudar o comportamento assintótico do valor esperado da covariância do erro de estimação $\mathcal{E}\{W(k)\}$, no sentido em que $\mathcal{E}\{W(k)\}$ seja limitado.

Por outro lado, possivelmente por incidência de erros nos termos que introduzem ruído ao sistema, no instante $k$ a covariância do erro de estimação verdadeira $W(k)$ não coincida com a covariância do erro de estimação calculada $P(k)$. Uma questão fundamental que surge é a divergência exponencial de $W(k)$, sendo de grande interesse 
o estudo de condições para obter limitação para $\mathcal{E}\{W(k)\}$ sempre que $\mathcal{E}\{P(k)\}$ for limitada.

Abordamos dois conceitos de estabilidade, a estabilidade com relação a condição inicial (c.r. $\Psi$ ) como em [7] e [10] ${ }^{1}$, esta noção de estabilidade requer que o valor esperado da covariância do erro de estimação seja limitada para incorreções em $\Sigma$. A segunda é uma noção relativamente mais usual de estabilidade de FK, no sentido de existência de limitação do valor esperado da covariância quando existe perturbações em ambos $E_{i}$ e $\Sigma$ do modelo nominal, como também abordada em [7] e [10].

Num primeiro momento, assumimos $\Sigma \neq \Psi$ e mostramos que detetabilidade estocástica e controlabilidade fraca ${ }^{2}$ constituem condições suficientes para que $\mathcal{E}\{W(k, E, \Psi)\}$ possua limitante superior para qualquer $k \geq 0$, obtendo assim estabilidade c.r $\Psi$. Consideramos um resultado mais geral, acrescentando erro no termo do ruido aditivo de estado, isto é, $E_{i} \neq B_{i}$, além de assumir erro na condição inicial e quando considerada uma desigualdade envolvendo o valor esperado de $W(k)$ (veja a Conjectura 1 , no Capítulo 5) e para o caso particular em que a cadeia de Markov possui distribuição inicial equilibrada (veja a Suposição 1 no Capítulo 5), obtemos um resultado análogo, a estabilidade do FK no sentido usual. Neste contexto, apresentamos resultados parciais, baseados numa conjectura e em uma hipótese adicional na cadeia de Markov, que merecem estudos futuros mais detalhados.

\subsection{Notações}

Nesta seção apresentam-se algumas notações para referência posterior.

Seja $\mathbb{R}^{n}$ o espaço euclidiano n-dimensional. Denotamos os números reais positivos por $\mathbb{R}_{+}$, os números naturais por $\mathbb{N}$ e seja $\mathbb{S}=\{0,1,2, \ldots, N\}$ um subconjunto de $\mathbb{N}$. Denota-se $\mathcal{M}^{r, s}$ (respectivamente $\mathcal{M}^{r}$ ), o espaço linear normado formado pelas matrizes reais $r \times s$ (respectivamente $r \times r$ ). Seja $\mathcal{M}^{r 0}$ o cone convexo formado pelas matrizes semi-definida positivas $\left\{U \in \mathcal{M}^{r}: U=U^{\prime} \geq 0\right\}$, onde $U^{\prime}$ denota a transposta de $U$. Para $U, V \in \mathcal{M}^{r}, U \geq V$ denota $U-V \in \mathcal{M}^{r 0}$. Seja $\mathcal{H}^{r, s}$ o espaço linear normado formado pelas sequências de matrizes $H_{i} \in \mathcal{M}^{r, s}, i \in \mathbb{S}$. Para $U, V \in \mathcal{H}^{r, s}, U+V=$ $\left\{U_{i}+V_{i}, \quad i \in \mathbb{S}\right\}, U \geq V$ denota $U_{i} \geq V_{i}$ para todo $i \in \mathbb{S}$ e similarmente para qualquer operação envolvendo termos pertencentes a $\mathcal{H}^{r, s}$. Denota-se a matriz identidade usual por II e o operador $1_{\{\cdot\}}$ representa a função indicadora. Respectivamente para os espaços

\footnotetext{
${ }^{1}$ A literatura utiliza o termo KF stable with respect to (w.r.t), aqui fazemos uso da terminologia em português, KF estável com relação a, (c.r).

${ }^{2}$ Por favor, veja definições nas Seções 2.4 e 3.1 respectivamente.
} 
$\mathcal{M}^{r}$ e $\mathcal{H}^{r}$, adotamos as normas abaixo ${ }^{3}$

$$
\begin{aligned}
\left\|A_{i}\right\|_{2} & =\sqrt{\rho\left(A_{i} A_{i}^{H}\right)}, i \in \mathbb{S} \\
\|A\| & =\max _{i \in \mathbb{S}}\left\{\left\|A_{i}\right\|_{2}\right\}
\end{aligned}
$$

Para uma dada matriz $P \in \mathcal{M}^{N}$ e $V \in \mathcal{H}^{r, n}$, introduzimos o operador $\mathcal{T}_{V}: \mathcal{H}^{r 0} \rightarrow$ $\mathcal{H}^{n 0}$ por

$$
\mathcal{T}_{V, i}(U)=\sum_{j=1}^{N} p_{j i} V_{j} U_{j} V_{j}^{\prime}, \quad i=1, \ldots, N,
$$

o qual é abordado em algumas definições. Os termos da matriz $P$ são representados por $p_{i j}$ e $\mathcal{T}_{V}$ é linear em seus argumentos. Denota-se $\mathcal{T}^{0}(U)=U$ e quando $\mathcal{T}: \mathcal{H}^{n 0} \rightarrow \mathcal{H}^{n 0}$, define-se $\mathcal{T}^{k}$ recursivamente por $\mathcal{T}^{k}(U)=\mathfrak{T}\left(\mathcal{T}^{k-1}(U)\right)$ para $k=1,2, \ldots$

$\operatorname{Sejam} \mathcal{A}$ e $\mathcal{J}$ variáveis aleatórias. Denotamos o valor esperado de $\mathcal{A}$ como $\mathcal{E}\{\mathcal{A}\}$, e o valor esperado condicional por $\mathcal{E}\{\mathcal{A} \mid \mathcal{J}\}$.

\subsection{Organização do texto}

Organizamos o texto da seguinte forma. No Capítulo 2 apresentamos alguns conceitos e resultados preliminares que introduzem o problema e serão utilizados ao longo dos capítulos seguintes. Na Seção 2.1 apresentam-se uma ideia geral do contexto de sistemas lineares e respectivas noções de estabilidade e ainda algumas propriedades usuais de matrizes e valor esperado; na Seção 2.2 apresentamos a noção de cadeia de Markov; na Seção 2.3 temos a motivação e o conceito de SLSM e finalmente na Seção 2.4 introduzimos o filtro de Kalman. No Capítulo 3 introduzimos na Seção 3.1 a definição de w-controlabilidade e apresentamos alguns resultados relevantes. No quarto capítulo, temos o tratamento da estabilidade com relação a condição inicial, mostra-se que w-controlabilidade e s-detetabilidade são condições suficientes para estabilidade c.r $\Psi$ do FK. No Capítulo 5 é feita a definição usual de estabilidade do FK, são obtidos resultados similares ao caso de estabilidade c.r $\psi$ e exemplos numéricos. Finalmente no Capítulo 6 tem-se as conclusões.

\footnotetext{
${ }^{3} A_{i}^{H}$ representa a matriz transposta conjudada de $A_{i}$.
} 


$\frac{-1}{2}$

\section{Conceitos e Resultados Preliminares}

Neste capítulo apresentamos na Seção 2.1, de forma breve, diferentes tipos de sistemas dinâmicos, assim como a motivação para o estudo destes sistemas, de forma a induzir a compreensão do comportamento dos sistemas lineares com saltos Markovianos. Na Seção 2.2 definimos e abordamos as principais propriedades da cadeia de Markov. Na Seção 2.3 apresentamos uma motivação, introduzimos os SLSMs e discutimos alguns resultados básicos. Na Seção 2.4 é realizada as definições de FK, termos auxiliares e resultados fundamentais para o estudo de estabilidade.

\subsection{Sistemas Lineares}

Em muitas situações práticas podem ser precisos os valores dos estados de um sistema dinâmico, contudo dispor-se apenas de medidas indiretas, dadas por um modelo que apresenta a variável de saída do sistema em função da variável de estado e um ruído de observação num instante de tempo $k \geq 0$, representado da seguinte forma

$$
y(k)=g(x(k), v(k)),
$$

sendo o vetor $y$ a medida (mais comumente chamado de saída), o vetor $x$ o estado, o vetor $v$ usualmente representando um ruído de observação e $g(\cdot)$ uma função. Em situações como esta surgem os observadores (estimadores da variável de estado. Neste trabalho abordamos a estimação da variável de estado em função da variável observada) para sistemas, os quais essencialmente constituem-se em um outro sistema dinâmico 
na forma

$$
\hat{x}(k+1)=h(y(k), \hat{x}(k)),
$$

em que a função $h(\cdot)$ é tipicamente projetada de forma a minimizar algum critério que aproxime a estimação do estado $\hat{x}$ de $x$. Em conexão com a sua importância e diversas aplicações, a pesquisa na área é bastante ativa, veja [13], [20], [31] e [39], apenas para mencionar alguns artigos em diferentes áreas.

Na implementação de um estimador, um sério problema que pode surgir consiste na instabilidade - informalmente, seria a situação na qual $\hat{x}$ "explode" para o infinito e para agravar a situação, mesmo observadores ótimos podem resultar instáveis, tornando a questão ainda mais importante. Esta "explosão" gera problemas numéricos e saturações, afetando tanto implementações computacionais quanto físicas.

Considere o sistema linear a tempo discreto

$$
\Phi: \begin{cases}x(k+1) & =A x(k)+B w(k) \\ y(k) & =C x(k)+D v(k) \\ x_{0} & =x_{0} \sim N(\sigma, \Psi)\end{cases}
$$

sendo $x \in \mathbb{R}^{n}$ o estado, $y \in \mathbb{R}^{n}$ a saída (aqui entendida como medições disponíveis) e $u \in \mathbb{R}^{r}$ o controle; admite-se que $v$ e $w$ são vetores aleatórios com distribuição $N(0, I)$.

Definição 2.1. Diz-se que A é estável se, para cada $z_{0} \in \mathbb{R}^{n}$,

$$
\lim _{k \rightarrow \infty}\|z(k)\|^{2}=0
$$

sendo $z(k+1)=A z(k)$.

A definição de estabilidade acima é usual para sistemas lineares e para esta classe de sistemas é equivalente a outras noções, como a de estabilidade exponencial, veja $[27]$;

Definição 2.2. Diz-se que o sistema é estável no sentido Exponencial Quadrático Médio (EEQM), se existirem constantes $0<\alpha<1$ e $\beta>0$, tais que para qualquer condição inicial $x_{0}$ e distribuição inicial $\mu_{0}$

$$
\mathcal{E}\left[\|x(k)\|^{2}\right] \leq \beta \alpha^{k}\|x(0)\|^{2}, \quad k \geq 0
$$

Por outro lado, com a necessidade da implementação de estimadores, surgem as noções de estabilidade dos filtros, no caso em questão, a estabilidade do FK para sistemas lineares discretos. Observemos que as noções de estabilidade do filtro e do sistema possuem abordagens distintas. As definições de estabilidade do FK para sistemas lineares discretos são consideradas como em [6]. 
Definição 2.3. (Estabilidade do FK para SLD) Dizemos que o FK é estável, se para cada $B \in \mathcal{H}^{n, r}$ e $\Psi \in \mathcal{M}^{n 0}$, existe $\bar{X}$ tal que $\mathcal{E}\left\{\tilde{x}(k) \tilde{x}(k)^{\prime}\right\} \leq \bar{X}$.

Para SLSMs, como veremos nos próximos capítulos, o aparecimento de termos com comportamento estocástico, induz a necessidade de introduzir ferramentas estatísticas para obtenção de conclusões sobre estabilidade do estimador. A qual é considerada em termos da trajetória do valor esperado.

\subsubsection{Propriedades usuais}

Destacamos aqui algumas propriedades usuais de matrizes e valor esperado, que serão consideradas no decorrer do texto.

Para quaisquer $A, B, C \in \mathcal{M}^{r, s}$ e $\mathcal{X}, \mathcal{G}, \mathcal{J}$ variáveis aleatórias, tem-se as seguintes propriedades para o valor esperado:

i) Se $A \geq B$, então $E\{A\} \geq E\{B\}$.

ii) Se $A \geq B$, então $\mathcal{E}\left\{C A C^{\prime}\right\} \geq \mathcal{E}\left\{C B C^{\prime}\right\}$.

iii) Linearidade : $\mathcal{E}\{(\alpha \mathcal{X}+\beta \mathcal{G}) \mid \mathcal{J}\}=\alpha \mathcal{E}\{(\mathcal{X}) \mid \mathcal{J}\}+\beta \mathcal{E}\{\mathcal{G}) \mid \mathcal{J}\}, \quad \alpha, \beta \in \mathbb{R}$.

iv) Independência : Se $\mathcal{X}, \mathcal{G}$ e $\mathcal{J}$ são variáveis aleatórias independentes, então $\mathcal{E}\{X \mathcal{G} \mid$ $\mathcal{\partial}\}=\mathcal{E}\{X \mid \mathcal{J}\} \mathcal{E}\{\mathcal{G} \mid \mathcal{J}\}$.

v) $\mathcal{E}\{X\}=\mathcal{E}\{\mathcal{E}\{X \mid \mathcal{G}\}\}$.

\subsection{Cadeia de Markov}

Para melhor entender os sistemas lineares com saltos Markovianos, considere um sistema que apresenta mais de um modo de operação. O sistema altera seu modo de operação de acordo com uma cadeia de Markov, isto é, a probabilidade dele passar de um modo de operação a outro depende apenas do seu modo atual. Considere ainda que sejam conhecidas todas estas probabilidades de transição de um modo de operação $i$ para $j$. A Figura 2.1 apresenta um sistema obedecendo uma cadeia de Markov com três modos, onde os círculos representam os diferentes modos de operação e os arcos com setas são as respectivas probabilidades de transição. A teoria de processos Markovianos compreende uma importante parte da teoria de processos estocásticos, este tema tem destaque em diversas aplicações, como em física, biologia, ciências sociais, engenharias e economia. 


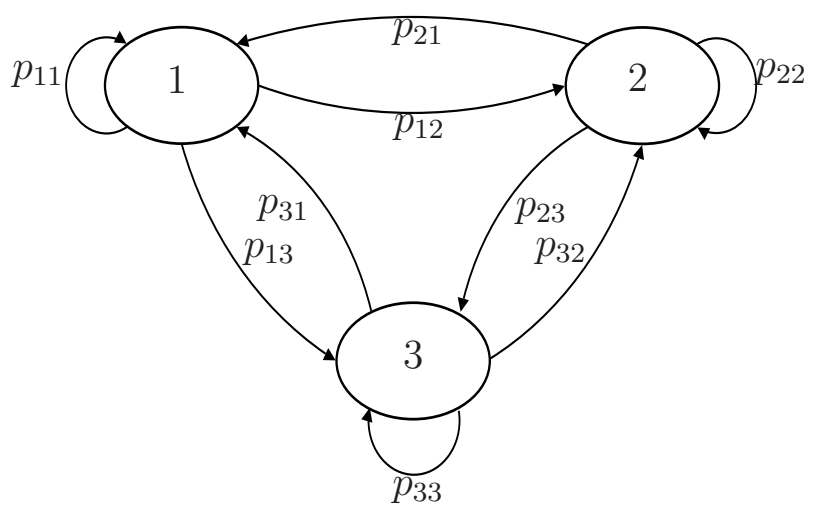

Figura 2.1: Representação da cadeia de Markov com três estados.

Formalmente, considera-se $\Omega$ um espaço amostral, $P$ a medida de probabilidade sobre este espaço e um processo estocástico ${ }^{1} \theta=\left[\theta_{n}, \quad n \in \mathbb{N}\right]$ com espaço de estado enumerável $\Theta$, isto é, para cada $n \in \mathbb{N}$ e $\omega \in \Omega, \theta_{n}(\omega)$ é um elemento de $\Theta$, veja [5].

Definição 2.4. O processo estocástico $\theta=\left[\theta_{n}: n \in \mathbb{N}\right]$ é chamado uma cadeia de Markov desde que

$$
\operatorname{Pr}\left\{\theta_{n+1}=j \mid \theta_{0}, \ldots, \theta_{n}\right\}=\operatorname{Pr}\left\{\theta_{n+1}=j \mid \theta_{n}\right\}, \quad \forall j \in E, \quad n \in \mathbb{N} .
$$

Denota-se uma cadeia de Markov por $\{\theta(k), \quad k \geq 0\}$. Neste trabalho consideramos o espaço de estado tomando valores num conjunto finito $\mathbb{S}=\{1,2, \ldots, N\}$.

Para cada estado existe uma probabilidade, as quais podem ser dispostas em um vetor $\pi$, denominado vetor de probabilidades de estados (para distingui-las das probabilidades de transição). A conexão com a matriz de probabilidade é como segue

$$
\pi(0)=\pi_{0} \text { e } \pi(r)=\pi(r-1) \mathbf{P}, \quad 1 \leq r \leq N,
$$

onde $\mathbf{P}=p_{i j}=\operatorname{Pr}\{\theta(k+1)=j \mid \theta(k)=i\}$ é a matriz de probabilidade de transição da cadeia de Markov. Um outro ponto que destacamos é a distribuição equilibrada da cadeia.

Definição 2.5. Diz-se que a Cadeia de Markov está equilibrada no instante $t$ quando $\operatorname{Pr}\left(\theta_{t}=i\right)=\operatorname{Pr}\left(\theta_{t+\tau}=i\right), \forall \tau=1,2, \ldots, \forall i \in \mathbb{S}$.

\footnotetext{
${ }^{1}$ Um processo estocástico é definido como uma coleção de variáveis aleatórias $X(t)$ indexadas por um parâmetro $t$ pertencente a um conjunto $T$, frequentemente tomado para ser o conjunto dos números naturais $\mathbb{N}$.
} 


\subsection{Os SLSMs}

Resumidamente, os SLSMs são sistemas estocásticos cuja dinâmica apresenta mudanças abruptas em instantes específicos e comportam-se como sistemas lineares nos demais instantes. As mudanças abruptas na dinâmica são chamados saltos, os quais se referem à alteração de parâmetros distintos do sistema que ocorrem de acordo com uma cadeia de Markov. Desta forma, a mudança para outro modo de operação ocorre de acordo com certa probabilidade, dependente apenas do modo em que o sistema se encontra no instante atual.

Com o objetivo de ilustrar e motivar a definição de SLSM, consideramos um exemplo simples e aplicado em diversas situações, o chamado sistema de controle de um pêndulo invertido equilibrado sobre uma base móvel semelhante às utilizadas por pequenos robôs móveis, veja [33]. O modelo encontra-se descrito na Figura $2.2^{2}$, em que $M$ é a massa da base, $H$ e $V$ são respectivamente as forças horizontal e vertical exercidas pelo carro sobre o pêndulo. Consideramos que existem falhas no rolamento, de tal forma que há um modo normal de operação e uma modo com "falha", no qual as contantes de atrito são maiores, se a mudança entre os dois modos ocorrer de acordo com uma probabilidade que dependa apenas do estado atual, podemos associar cada um dos estados a uma cadeia de Markov. Dessa forma, para o primeiro modo, o sistema do pêndulo invertido pode ser descrita como

$$
x(k+1)=A_{\theta(1)} x(k)+B_{\theta(1)} w(k)
$$

e o segundo modo como

$$
x(k+1)=A_{\theta(2)} x(k)+B_{\theta(2)} w(k) .
$$

Nesta perspectiva, define-se um sistema linear com saltos Markovianos a tempo discreto, descrito em um espaço de probabilidade $\left(\Omega, F, F_{k}, \mathbf{P}\right)$ por:

$$
\Phi_{M}: \begin{cases}x(k+1) & =A_{\theta(k)} x(k)+B_{\theta(k)} w(k) \\ y(k) & =C_{\theta(k)} x(k)+D_{\theta(k)} v(k) \\ x(0) & =x_{0}, \theta(0)=\theta_{0}\end{cases}
$$

para $k=0,1, \ldots$ sendo o par $(x(k), \theta(k))$ o estado do sistema, com $x \in \mathbb{R}^{n}$ a variável contínua e $\theta \in \mathbb{S}=\{1,2, \ldots, N\}$ a variável discreta do estado, $y \in \mathbb{R}^{n}$ a variável observada, $w \in \mathbb{R}^{p}$ e $v \in \mathbb{R}^{q}$ ruídos de observação aleatórios com distribuição $N(0, I) ; \theta(k)$ é o estado de uma cadeia de Markov discreta no tempo, com espaço de estado finito com matriz de probabilidade de transição $\mathbf{P}=\left[p_{i j}\right] \in \mathcal{M}^{N}$, tal

\footnotetext{
${ }^{2}$ Figura extraída de [4].
} 
que $p_{i j}:=\operatorname{Pr}\{\theta(k+1)=j \mid \theta(k)=i\}$ é a probabilidade do sistema passar do modo de operação $i$ para $j$, portanto deve satisfazer $p_{i j} \geq 0$ para $i, j \in \mathbb{S}$ e para cada $i$, $\sum_{j=1}^{N} p_{i j}=1$. Toma-se $\pi(k)=P\{\theta(k)=i\}$, sempre que $\theta=i, i \in \mathbb{S}$. Neste trabalho há contextos em que se lida com as observações $\Theta_{k}=\{x(0), \theta(0), \ldots, \theta(k)\}$ ou $\mathfrak{F}_{k}=\{y(0), \theta(0), \ldots, y(k), \theta(k)\}$. Sendo $A_{i} \in \mathcal{M}^{n}, B_{i} \in \mathcal{M}^{r, n}, C_{i} \in \mathcal{M}^{r, n}$ e $D_{i} \in \mathcal{M}^{r, q}$, com $D_{i} D_{i}^{\prime}>0$ (ruído não singular).

As aplicações para SLSM são diversas, dentre as quais citamos, aeronáutica [3], modelos macroeconômicos [23] e receptores térmicos [43]. Muitos resultados teóricos estão presentes na literatura de SLSM, dentre os quais destacamos [12], [13], [14], [16],[17], [18], [22], [28], [30], [35] e [41]. Alguns aspectos interessantes sobre como SLSM generalizam SLD podem ser encontrados em [8] e [24].

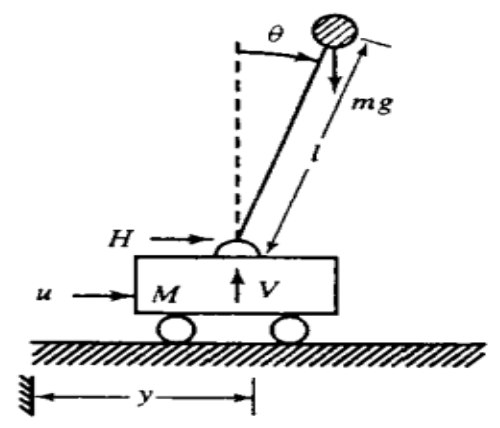

Figura 2.2: Modelo físico de um Sistema de Suspensão com Saltos Markovianos

\subsection{Filtro de Kalman para Sistemas Lineares com Sal- tos Markovianos}

O FK é um dos filtros mais conhecidos e aplicados em sistemas dinâmicos. É ótimo em diferentes sentidos, linear, recursivo e eficiente computacionalmente, veja por exemplo [1] e [31]. Além disso, apresenta relações estruturais com o sistema original, que permite caracterizar limites superiores para o valor esperado da covariância do erro de estimação e outros aspectos fundamentais, baseados em modelos do sistema e ruído. Nessa linha, sabe-se que o FK para um sistema linear variante no tempo apresenta covariância do erro limitada, quando o sistema é estocasticamente detetável, veja [10]. A priori, um conhecimento exato das propriedades do filtro para cada instante de tempo $k \geq 0$, possibilita a verificação de detetabilidade e então inferir propriedades sobre a 
covariância do erro de estimação, veja [36], [42] e [44].

Neste trabalho adotamos a predição com um passo, isto é, $\hat{x}(k+1)=\mathcal{E}\left\{x(k+1) \mid \mathcal{F}_{k}\right\}$. A seguir apresentamos a formalização desse filtro, observando que o filtro de Kalman para SLSM, com a observação dos estados $\theta$ de Markov, é equivalente ao FK para sistemas lineares discretos variantes no tempo, veja [19] e [30].

Para estimação do estado discreto $x(k)$ consideramos o sistema $\Phi_{M}$, apresentado em (2.2), escrito com a substituição dos valores reais pelos valores nominais:

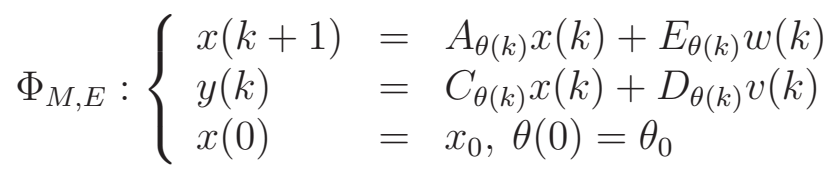

$\operatorname{com} \mathcal{E}\left\{\tilde{x}(k) \tilde{x}(k)^{\prime}\right\}=\Sigma$.

Consideramos :

i) O ruído $w(k)$ tenha média nula, covariância igual a $\mathbb{I}$ e não seja correlacionado entre si, ou seja $\mathcal{E}\left\{w(k) w(j)^{\prime}\right\}=0, k \neq j$.

ii) O ruído $v(k)$ tenha média nula e covariância igual a $\mathbb{I}, \mathcal{E}\left\{v(k) v(j)^{\prime}\right\}=0, k \neq j$ e $\mathcal{E}\left\{x(k) v(k)^{\prime}\right\}=0, k \geq 0$.

iii) O estado não seja correlacionado ao ruído, isto é, $\mathcal{E}\left\{x(k) w(k)^{\prime}\right\}=0, k \geq 0$.

iv) Os ruídos $v(k)$ e $w(k)$ sejam descorrelacionados, ou seja, $\mathcal{E}\left\{w(k) v(k)^{\prime}\right\}=0, k \geq 0$.

Para construção do filtro recursivo de Kalman assumimos ainda que no instante $k$ sejam conhecidas as observações do estado de Markov e a saída do sistema até o instante atual, ou seja, $\mathcal{F}_{k}=[y(0), \theta(0), y(1), \theta(1), \ldots, y(k), \theta(k)]$, como em [13] e [20]. O filtro é obtido recursivamente, considerando

$$
\hat{x}(k)=\mathcal{E}\left\{x(k) \mid \mathfrak{F}_{k-1}\right\},
$$

dado por $\hat{x}(0)=0 \mathrm{e}$

$$
\hat{x}(k+1)=A_{\theta(k)} \hat{x}(k)+L_{k}\left[y(k)-C_{\theta(k)} \hat{x}(k)\right],
$$

onde o ganho de Kalman

$$
L_{k}=A_{\theta(k)} P(k) C_{\theta(k)}^{\prime}\left[C_{\theta(k)} P(k) C_{\theta(k)}^{\prime}+D_{\theta(k)} D_{\theta(k)}^{\prime}\right]^{-1},
$$

é calculado via a equação recursiva de Riccati

$$
\begin{aligned}
P(k+1) & =A_{\theta(k)}\left[P(k)-P(k) C_{\theta(k)}^{\prime}\left(C_{\theta(k)} P(k) C_{\theta(k)}^{\prime}+D_{\theta(k)} D_{\theta(k)}^{\prime}\right)^{-1}\right. \\
& \left.\times C_{\theta(k)} P(k)\right] A_{\theta(k)}^{\prime}+E_{\theta(k)} E_{\theta(k)}^{\prime},
\end{aligned}
$$


considerando $P(0)=\Sigma$.

Para uma sequência de ganhos $L_{k} \in \mathcal{M}^{n, r}, k \in \mathbb{N}$, considere o erro de estimação

$$
\tilde{x}(k)=x(k)-\hat{x}(k),
$$

o qual pode ser escrito como

$$
\tilde{x}(k+1)=\left(A_{\theta(k)}-L_{k} C_{\theta(k)}\right) \tilde{x}(k)+B_{\theta(k)} w(k)-L_{k} D_{\theta(k)} v(k) .
$$

A covariância do erro real $W(k, B, \Psi) \in \mathcal{R}^{n 0}$ é obtida recursivamente por $W(0, B, \Psi)=$ $\Psi, \Psi \in \mathcal{M}^{n 0} \mathrm{e}$

$$
\begin{aligned}
W(k+1, B, \Psi) & =\left(A_{\theta(k)}-L_{k} C_{\theta(k)}\right) W(k, B, \Psi)\left(A_{\theta(k)}-L_{k} C_{\theta(k)}\right)^{\prime} \\
& +L_{k} D_{\theta(k)} D_{\theta(k)}^{\prime} L_{k}^{\prime}+B_{\theta(k)} B_{\theta(k)}^{\prime} .
\end{aligned}
$$

A solução homogênea de (2.7) é como segue

$$
\begin{aligned}
W_{h}(k, l, \Psi) & =\left(A_{\theta(k)}-L_{k} C_{\theta(k)}\right) \ldots \times\left(A_{\theta(l)}-L_{l} C_{\theta(l)}\right) \\
& \times \Psi\left(A_{\theta(l)}-L_{l} C_{\theta(l)}\right)^{\prime} \ldots \times\left(A_{\theta(k)}-L_{k} C_{\theta(k)}\right)^{\prime}, \quad k \geq l \geq 0 .
\end{aligned}
$$

A critério de organização dos termos e visando facilitar a notação, rescrevemos (2.7) em função da solução homogênea:

$$
W(k+1, B, \Psi)=W(k+1,0, \Psi)+\sum_{l=1}^{k} W_{h}\left(k, l, \Upsilon_{l-1}(B)\right)
$$

$\operatorname{com} \Upsilon_{k}(B)=L_{k} D_{\theta(k)} D_{\theta(k)}^{\prime} L_{k}^{\prime}+B_{\theta(k)} B_{\theta(k)}^{\prime}$

Considere a seguinte definição

Definição 2.6. Recursivamente define-se

$$
X(k+1, B, \Psi)=A_{\theta(k)} X(k, B, \Psi) A_{\theta(k)}^{\prime}+B_{\theta(k)} B_{\theta(k)}^{\prime},
$$

$\operatorname{com} X(0, B, \Psi)=\Psi$.

Rescrevemos (2.10) em função da solução homogênea

$$
X(k+1, B, \Psi)=X(k+1,0, \Psi)+\sum_{l=1}^{k} X_{h}\left(k, l, B_{\theta(l-1)} B_{\theta(l-1)}^{\prime}\right),
$$

com

$$
X_{h}\left(k, l, B_{\theta(l-1)} B_{\theta(l-1)}^{\prime}\right)=A_{\theta(k)} A_{\theta(k-1)} \ldots A_{\theta(l)} B_{\theta(l-1)} B_{\theta(l-1)}^{\prime} A_{\theta(l)}^{\prime} \ldots A_{\theta(k-1)}^{\prime} A_{\theta(k)}^{\prime}
$$


Observação 1. Observemos que a covariância do erro de estimação e a covariância do estado são definidos em função da cadeia de Markov $\theta(k), k \in \mathbb{S}$ e portanto formam processos estocásticos, dessa forma trabalharemos com os valores esperados $\mathcal{E}\left\{X(k) \mid \mathcal{F}_{k}\right\}$ $e \mathcal{E}\left\{W(k) \mid \mathcal{F}_{k}\right\}$.

Consideramos a formulação de detetabilidade estocástica de [10].

Definição 2.7. Diz-se que $(A, \mathbf{P})$ é estocasticamente estável (s-estável) se, para cada $V \in \mathcal{M}^{n, r}$, existe $\bar{V} \in \mathcal{M}^{r 0}$ tal que $\sum_{k=0}^{\infty} \mathcal{T}_{A}^{k}(V) \leq \bar{V}$. Diz-se que $(A, C, \mathbf{P})$ é estocasticamente detetável, se existe $K \in \mathcal{H}^{n, r}$ tal que $(A+K C, \mathbf{P})$ seja s-estável.

A conexão entre a expressão em (2.10) e a definição de covariância do estado é como na proposição abaixo, veja [40].

Proposição 2.1. Considere a definição de $X(k, B, \Psi)$ dada em (2.10). Então

$$
\mathcal{E}\left\{x(k) x(k)^{\prime} \mid \mathcal{F}_{k}\right\}=X(k, B, \Psi) .
$$

Neste trabalho, a hipótese de limitação do valor esperado da covariância do erro de estimação para os valores nominais de $\Sigma$ e $E$ é de grande interesse. Esta questão foi abordada em [10], onde se mostra uma condição suficiente para limitação de $\mathcal{E}\{P(k)\}$. Exprimimos este resultado na seguinte proposição.

Proposição 2.2. Considere $P(k)$ como definida em (2.6) e assuma que $(A, C, \mathbf{P})$ seja estocasticamente detetável. Então, existe $\bar{P} \in \mathcal{M}^{r 0}$ tal que $\mathcal{E}\{P(k)\} \leq \bar{P}, k \geq 0$.

O lema seguinte mostra que a limitação de $\mathcal{E}\{P(k)\}$ é uma condição suficiente para limitação do valor esperado do ganho de Kalman.

Lema 2.1. Considere $P(k)$ definida em (2.6) e o ganho de Kalman $L_{k}$ como em (2.5). $S e(A, C, \boldsymbol{P})$ é estocasticamente detetável, então existe $M \in \mathcal{M}^{n 0}$ tal que $\mathcal{E}\left\{\left\|L_{k}\right\|\right\}<M$.

Demonstração: Temos por (2.5) que

$$
\begin{aligned}
\mathcal{E}\left\{\left\|L_{k}\right\|\right\} & \leq\|A\| \mathcal{E}\{\|P(k)\|\}\|C\|\left[\|C\| \mathcal{E}\{\|P(k)\|\}\left\|C^{\prime}\right\|+\left\|D D^{\prime}\right\|\right]^{-1} \\
& \leq\|A\| \bar{P} \|\left[\|C\| \bar{P}\left\|C^{\prime}\right\|+\left\|D D^{\prime}\right\|\right]^{-1}=M,
\end{aligned}
$$

concluindo a demonstração.

Observemos que sem a hipótese de limitação de $P(k)$, não se pode falar de limitação de $W(k, B, \Psi)$, uma vez que esta também se tornaria divergente, no sentido de manter crescimento ilimitado. Assumimos para todos os resultados nas seções seguintes que $(A, C, \mathbf{P})$ é estocáticamente detetável. A limitação de $\mathcal{E}\left\{\left\|L_{k}\right\|\right\}$ nos permite obter estimativas do comportamento de $W(k)$. 



\begin{tabular}{|l|c|}
\cline { 2 - 2 } & Capítulo \\
\cline { 2 - 2 } & 3 \\
\hline
\end{tabular}

\section{W-controlabilidade}

Neste capítulo apresentamos na Seção 3.1 o conceito de controlabilidade fraca (wcontrolabilidade) e é realizada a construção da matriz de controlabilidade para SLSMs. Discutimos as principais características do conceito em diferentes contextos já abordados em pesquisas na área. Na Seção 3.3 são apresentados alguns resultados relevantes para os próximos capítulos.

\subsection{Controlabilidade para SLSM}

A estrutura de entrada e saída pode influenciar de forma significativa os meios disponíveis para controle. Um conceito fundamental que caracteriza implicações na dinâmica da estrutura de entrada e saída é a controlabilidade. Para um sistema linear discreto e invariante no tempo, o par $(A, E)$ (com $A$ e $E$ as matrizes representadas no sistema dado em 2.2) é controlável se para qualquer estado inicial $x(0)=x_{0}$ e para qualquer estado final $x_{f}$ existir uma entrada $u(t)$ que transfere o estado de $x_{0}$ para $x_{f}$ em tempo finito. A definição requer apenas que se possa "mover"qualquer estado inicial no espaço de estados para qualquer estado final em tempo finito. Não há restrições quanto à trajetória a ser seguida, nem quanto à magnitude da entrada, veja [34].

A controlabilidade para SLSMs tem sido abordada em pesquisas recentes. Em [9] é feita a introdução das matrizes de w-controlabilidade, análogas as matrizes de 
controlabilidade usuais de SLD. Os resultados aqui apresentados consideram a definição de w-controlabilidade ${ }^{1}$ introduzida nesta mesma referência.

Considere o sistema $\Phi_{M, E}$, introduzido em (2.3). Definem-se as matrizes $S_{i}(k) \in$ $\mathcal{M}^{n 0}$ por

$$
S_{i}(k+1)=\mathcal{E}\left\{x(k) x(k)^{\prime} 1_{\{\theta(k)=i\}}\right\}, \quad i=1, \ldots, N
$$

Pode-se representar $S_{i}(k)$ em termos do operador $\mathcal{T}_{A, i}$, definido em (1.1), como feito abaixo

$$
\begin{cases}S_{i}(k+1) & =\mathcal{T}_{A, i}(S(k))+\mathcal{T}_{E, i}(\mathbb{I}), k=0,1, \ldots, i \in \mathbb{S} \\ S_{i}(0) & =0, i \in \mathbb{S}\end{cases}
$$

ou equivalentemente, $\operatorname{com} Q=\mathcal{T}_{E}(\mathbb{I})$

$$
S(k)=\mathcal{T}_{A}^{k}(S(0))+\sum_{l=0}^{k-1} \mathcal{T}_{A}^{l}(Q)=\sum_{l=0}^{k-1} \mathcal{T}_{A}^{l}(Q), \quad k=0,1, \ldots
$$

Veja [15] para uma prova. Considera-se a matriz de w-controlabilidade

$$
\mathcal{C}_{i}(m)=\left[S_{i}(1) \vdots S_{i}(2) \vdots \ldots \vdots S_{i}(n)\right], \quad n \in \mathbb{N}, \quad i=1, \ldots, N
$$

com $S_{i}(k)$ dado em (3.2).

Similarmente ao caso de SLDs, a matriz de w-controlabilidade pode ser limitada em termos de $n$ e $N$, veja [9, Corollary 1].

Observação 2. Quando $N=1$ e $\Sigma=0, \mathcal{C}_{i}(n)$ se reduz à matriz usual de controlabilidade para SLDs, veja [9].

A seguir apresentam-se os conceitos de controlabilidade fraca de [9].

Definição 3.1. Considere o sistema $\Phi_{M, E}$ e a coleção de matrizes $\mathfrak{C}_{i}(n), i \in \mathbb{S}$ apresentada em (3.4). Diz-se que $(A, E, \mathbf{P})$ é w-controlável se posto $\left(\mathfrak{C}_{i}\left(n^{2} N\right)=n, i \in \mathbb{S}\right.$.

A seguir apresenta-se o Lema 3.1 que traz um resultado fundamental para o estudo de estabilidade: a interpretação é que se um sistema $(A, E, \mathbf{P})$ for controlável então temos positividade de $S_{i}\left(n^{2} N\right)$, ou seja, o ruído excita todas as direções do sistema.

Lema 3.1. Considere o sistema $\Phi_{M, E}$ e a coleção de matrizes $\mathcal{C}_{i}(m)$ apresentada em (3.4),

$$
\operatorname{posto}\left(\mathfrak{C}_{i}\left(n^{2} N\right)\right)=n, \text { se e somente se, posto }\left(S_{i}\left(n^{2} N\right)\right)=n \text {. }
$$

\footnotetext{
${ }^{1} \mathrm{O}$ termo w-controlável se refere a controlabilidade fraca e a terminologia vem no inglês weak controllability.
} 
Demonstração: Temos de (3.4) que

$$
\mathcal{C}_{i}\left(n^{2} N\right) \mathcal{C}_{i}\left(n^{2} N\right)^{\prime}=\sum_{k=0}^{n^{2} N} S_{i}(k) S_{i}(k)^{\prime}
$$

Na sequência mostramos que

$$
S_{i}\left(n^{2} N\right) S_{i}\left(n^{2} N\right)^{\prime}>0 \Leftrightarrow \sum_{k=0}^{n^{2} N} S_{i}(k) S_{i}(k)^{\prime}>0 .
$$

1. (Condição suficiente) Segue direto que

$$
S_{i}\left(n^{2} N\right) S_{i}\left(n^{2} N\right)^{\prime}>0 \Rightarrow \sum_{k=0}^{n^{2} N} S_{i}(k) S_{i}(k)^{\prime}>0,
$$

portanto, de (3.5), temos

$$
\mathcal{C}\left(n^{2} N\right) \mathrm{C}^{\prime}\left(n^{2} N\right)>0
$$

2. (Condição necessária) A prova da condição necessária é feita por contradição. Assuma que seja válida a seguinte desigualdade

$$
\sum_{k=1}^{n^{2} N} S_{i}(k) S_{i}(k)^{\prime}>0
$$

e suponha que exista $v \in \mathbb{R}^{n}$ tal que $v^{\prime} S_{i}\left(n^{2} N\right) S_{i}\left(n^{2} N\right)^{\prime} v=0$. De (3.3), temos que

$$
\begin{aligned}
0 & =v^{\prime} S_{i}\left(n^{2} N\right) S_{i}\left(n^{2} N\right)^{\prime} v=v^{\prime}\left[\sum_{l=0}^{n^{2} N-1} \mathcal{T}_{A, i}^{l}(Q)\right]\left[\sum_{l=0}^{n^{2} N-1} \mathcal{T}_{A, i}^{l}(Q)\right]^{\prime} v \\
& \geq v^{\prime}\left[\sum_{l=0}^{k-1} \mathcal{T}_{A, i}^{l}(Q)\right]\left[\sum_{l=0}^{k-1} \mathcal{T}_{A, i}^{l}(Q)\right]^{\prime} v
\end{aligned}
$$

para qualquer $0 \leq k \leq n^{2} N-1$, onde empregamos o fato dos termos das somas serem não negativos. Temos portanto que

$$
0=v^{\prime}\left[\sum_{l=0}^{k-1} \mathcal{T}_{A, i}^{l}(Q)\right]\left[\sum_{l=0}^{k-1} \mathcal{T}_{A, i}^{l}(Q)\right]^{\prime} v=v^{\prime}\left[S_{i}(k) S_{i}(k)^{\prime}\right] v, \quad \forall \quad 0 \leq k \leq n^{2} N-1,
$$

contradizendo (3.6), portanto

$$
v^{\prime}\left[S_{i}\left(n^{2} N\right) S_{i}\left(n^{2} N\right)^{\prime}\right] v>0, \quad \forall v \in \mathbb{R}^{n},
$$

completando a prova. 


\subsection{Exemplos Numéricos}

Como ilustração, apresentamos os seguintes exemplos de SLSMs w-controláveis e não w-controláveis respectivamente, fazendo uso das Definições 3.1 e do Lema 3.1.

Exemplo 3.1. ( Sistema w-controlável) Considere um sistema como $\Phi_{M, E}$, com os seguintes dados

$$
\begin{gathered}
A_{1}=\left[\begin{array}{cc}
0.9 & 1 \\
0 & 0
\end{array}\right], A_{2}=\left[\begin{array}{cc}
0.9 & 0 \\
0 & 1
\end{array}\right], E_{1}=0, E_{2}=\left[\begin{array}{ll}
0 & 0 \\
0 & 1
\end{array}\right], C_{1}=C_{2}=\left[\begin{array}{ll}
1 & 0
\end{array}\right], \\
D_{1}=D_{2}=I_{3}, \quad \boldsymbol{P}=\left[\begin{array}{ll}
0.1 & 0.9 \\
0.1 & 0.9
\end{array}\right], \pi(0)=\left[\begin{array}{ll}
0.5 & 0.5
\end{array}\right] \text { e } \Sigma=0 .
\end{gathered}
$$

Podemos verificar que

$$
\begin{aligned}
& \text { posto }\left(\mathrm{C}_{1}(8)\right)=\operatorname{posto}\left(\left[S_{1}(1) \vdots S_{1}(2) \vdots \ldots \vdots S_{1}(8)\right]\right) \\
& =\operatorname{posto}\left(\left[\begin{array}{ccccccccccccc}
0 & 0 & \vdots & 0 & 0 & \vdots & 0.0001 & 0 & \vdots & \ldots & \vdots & 0.0166 & 0 \\
0 & 0 & \vdots & 0 & 0.01 & \vdots & 0 & 0.0361 & \vdots & \ldots & \vdots & 0 & 0.2722
\end{array}\right]\right)=2 \text {, } \\
& \operatorname{posto}\left(\mathrm{C}_{2}(8)\right)=\operatorname{posto}\left(\left[S_{2}(1) \vdots S_{2}(2) \vdots \ldots \vdots S_{2}(8)\right]\right) \\
& =\operatorname{posto}\left(\left[\begin{array}{ccccccccccccc}
0 & 0 & \vdots & 0 & 0 & \vdots & 0.0001 & 0 & \vdots & \ldots & \vdots & 1.3408 & 0 \\
0 & 0 & \vdots & 0 & 0.01 & \vdots & 0 & 0.0361 & \vdots & \ldots & \vdots & 0 & 22.0461
\end{array}\right]\right)=2 \text {, }
\end{aligned}
$$

portanto, pela Definição 3.1, (A,E,P) é w-controlável.

Por outro lado,

$$
\begin{aligned}
& \operatorname{posto}\left(S_{1}(8)\right)=\text { posto }\left(\left[\begin{array}{cc}
0.0166 & 0 \\
0 & 0.2722
\end{array}\right]\right)=2, \\
& \operatorname{posto}\left(S_{2}(8)\right)=\text { posto }\left(\left[\begin{array}{cc}
1.3408 & 0 \\
0 & 22.0461
\end{array}\right]\right)=2,
\end{aligned}
$$

portanto pelo Lema 3.1 ( $A, E, \mathbf{P})$ é w-controlável.

Exemplo 3.2. (Sistema não w-controlável) Considere um sistema $\Phi_{M, E}$ como no Exemplo 3.1, com os seguintes dados

$$
A_{1}=A_{2}=\left[\begin{array}{cc}
0.9 & 0 \\
0 & 0
\end{array}\right] \text {. }
$$

Podemos verificar que

$$
\begin{aligned}
\operatorname{posto}\left(\mathrm{C}_{1}(8)\right) & =\operatorname{posto}\left(\left[S_{i}(1) \vdots S_{i}(2) \vdots \ldots\right.\right. \\
& =\operatorname{posto}\left(\left[\begin{array}{ccccccccc}
0 & 0 & \vdots & 0 & 0 & \ldots & \vdots & 0 & 0 \\
0 & 0 & \vdots & 0 & 0.01 & \ldots & \vdots & 0 & 0.01
\end{array}\right]\right)=1,
\end{aligned}
$$


portanto através das Definições 3.1, $(A, E, \mathbf{P})$ é não w-controlável.

Por outro lado

$$
\operatorname{posto}\left(S_{1}(8)\right)=\operatorname{posto}\left(\left[\begin{array}{cc}
0 & 0 \\
0 & 0.01
\end{array}\right]\right)=1,
$$

portanto pela Lema 3.1, (A,E, P) é não w-controlável.

\subsection{Positividade da covariância}

Os resultados seguintes dizem respeito as condições suficientes para positividade do valor esperado da covariância de $x(k)$ definida em $(2.10)$ e de $\tilde{x}(k)$ definida em $(2.7)$ nos instantes de tempo da forma $k=\ln ^{2} N, l \geq 1$. Este fato é de importância para obtenção de uma trajetória auxiliar na construção de limitantes para $\mathcal{E}\{W(k)\}$.

Lema 3.2. Considere um sistema como em $\Phi_{M, E}$, substituindo os valores reais pelos valores nominais e a covariância do estado $X$ definida em (2.10). Se $(A, E, \mathbf{P})$ é w-controlável então

$$
\mathcal{E}\left\{X\left(n^{2} N, E, \Sigma\right)\right\}>0
$$

Demonstração: Pela Proposição 2.1 temos que

$$
\begin{aligned}
\mathcal{E}\left\{X\left(n^{2} N, E, \Sigma\right)\right\} & \geq \mathcal{E}\left\{X\left(n^{2} N, E, \Sigma=0\right)\right\} \\
& =\mathcal{E}\left\{x\left(n^{2} N\right) x\left(n^{2} N\right)^{\prime} \mid \Sigma=0\right\}=\mathcal{E}\left\{x\left(n^{2} N\right) x\left(n^{2} N\right)^{\prime} \sum_{i=1}^{N} 1_{\theta(k)=i} \mid \Sigma=0\right\} \\
& =\sum_{i=1}^{N} \mathcal{E}\left\{x\left(n^{2} N\right) x\left(n^{2} N\right)^{\prime} 1_{\theta(k)=i} \mid \Sigma=0\right\}=\sum_{i=1}^{N} S_{i}\left(n^{2} N\right)>0
\end{aligned}
$$

e o lema está demonstrado.

O corolário a seguir trata do termo da covariância do estado dependente da matriz de ruído aditivo $E$.

Corolário 3.1. Considere o sistema $\Phi_{M, E}$ e a solução homogênea da covariância do estado $X_{h}$, como definido em (2.11). Se $(A, E, \mathbf{P})$ é w-controlável então

$$
\mathcal{E}\left\{\sum_{k=1}^{n^{2} N-1} X_{h}\left(n^{2} N-1, k, E_{\theta(k-1)} E_{\theta(k-1)}^{\prime}\right)\right\}>0 .
$$


Demonstração: Considere no Lema 3.2 o caso particular em que $\Sigma=0$, portanto por (2.11) tem-se

$$
\begin{aligned}
& \mathcal{E}\left\{\sum_{k=1}^{n^{2} N-1} X_{h}\left(n^{2} N-1, k, E_{\theta(k-1)} E_{\theta(k-1)}^{\prime}\right)\right\} \\
& \quad=\mathcal{E}\left\{X\left(n^{2} N, 0, \Sigma\right)\right\}+\mathcal{E}\left\{\sum_{k=1}^{n^{2} N-1} X_{h}\left(n^{2} N l-1, k, E_{\theta(k-1)} E_{\theta(k-1)}^{\prime}\right)\right\} \\
& \quad=\mathcal{E}\left\{X\left(n^{2} N, E, \Sigma\right)\right\}>0,
\end{aligned}
$$

concluindo a demonstração.

Observação 3. Observe que a validade do Corolário 3.1 depende apenas das matrizes $A_{i}$ e $E_{i}, i \in \mathbb{S}$ do sistema $\Phi_{M, E}$ definido em (2.2) e do tamanho do intervalo $\left[0, n^{2} N\right]$.

Estamos interessados que o valor esperado de $X(k)$ possua um limitante inferior uniforme para todos os instantes da forma $k=\ln ^{2} N, l \geq 1$. Os corolários abaixo tratam esta questão.

Corolário 3.2. Considere o sistema $\Phi_{M, E}$ e $X$ como definida em (2.10). Se $(A, E, \mathbf{P})$ é w-controlável, então existe $\mu>0$, tal que $\mathcal{E}\left\{X\left(n^{2} N, E, \Sigma\right)\right\} \geq \mu I$.

O corolário abaixo faz extensão do Corolário 3.2 para todo $k=\ln ^{2} N$, com $l>1$.

Corolário 3.3. Considere o sistema $\Phi_{M, E}$ e $X$ como definido em (2.10). Se $(A, E, \mathbf{P})$ é w-controlável, então

$$
\mathcal{E}\left\{X\left(\ln ^{2} N, E, \Sigma\right)\right\} \geq \mu I, \quad l \geq 1 .
$$

Demonstração: Desenvolvendo (3.2) recursivamente em termos de $\ln ^{2} N$ e $0 \leq k \leq$ $\ln ^{2} N$ como em (3.3) tem-se

$$
\begin{aligned}
S_{i}\left(\ln ^{2} N\right) & =\sum_{k=0}^{l n^{2} N-1} \mathcal{T}_{A, i}^{k}\left(\mathcal{T}_{E}(\mathbb{I})\right) \\
& =\sum_{k=0}^{n^{2} N-1} \mathcal{T}_{A, i}^{n^{2} N}\left(\mathcal{T}_{E}(\mathbb{I})\right)+\sum_{k=n^{2} N}^{l n^{2} N-1} \mathcal{T}_{A, i}^{k}\left(\mathcal{T}_{E}(\mathbb{I})\right) \\
& =S_{i}\left(n^{2} N\right)+\sum_{k=n^{2} N}^{l n^{2} N-1} \mathcal{T}_{A, i}^{k}\left(\mathcal{T}_{E}(\mathbb{I})\right)
\end{aligned}
$$

portanto, tomando a soma de $S_{i}\left(\ln ^{2} N\right)$ em (3.7) como a seguir e pelo Lema 3.2 obtém-se

$$
\sum_{k=0}^{N} S_{i}\left(\ln ^{2} N\right)=\sum_{k=0}^{N} S_{i}\left(n^{2} N\right)+\sum_{k=0}^{N}\left(\sum_{k=n^{2} N}^{l n^{2} N-1} \mathcal{T}_{A, i}^{k}\left(\mathcal{T}_{E}(\mathbb{I})\right)\right) \geq \mu I,
$$


$\log 0$

$$
\mathcal{E}\left\{X\left(\ln ^{2} N, E, \Sigma\right)\right\} \geq \mathcal{E}\left\{X\left(\ln ^{2} N, E, \Sigma=0\right)\right\}=\mathcal{E}\left\{\sum_{i=1}^{N} S_{i}\left(\ln ^{2} N\right)\right\} \geq \mu I
$$

como queríamos demonstrar.

Até o momento, obtemos condições para existência de uma limitação inferior para o valor esperado da covariância do estado $\mathcal{E}\left\{X\left(\ln ^{2} N\right)\right\}, l \geq 1$. Busca-se a extensão dos resultados anteriores para a covariância do erro de estimação $\mathcal{E}\{W(k)\}$, definida em (2.7). De fato a positividade de $\mathcal{E}\left\{X\left(\ln ^{2} N\right)\right\}$ implica a positividade de $\mathcal{E}\left\{W\left(\ln ^{2} N\right)\right\}, \quad l \geq 1$. Os próximos corolários fornecem a demonstração desta afirmação.

Corolário 3.4. Considere o sistema $\Phi_{M, E}$ e a covariância do erro de estimação, como definido em (2.7). Se (A,E, P) é w-controlável, então existe $\bar{\mu} \in \mathbb{R}_{+}$tal que

$$
\mathcal{E}\left\{W\left(n^{2} N, E, \Sigma\right)\right\} \geq \bar{\mu} I .
$$

Demonstração: A prova é feita por contradição. Suponha que exista $v \in \mathbb{R}^{n}$ tal que $v^{\prime} \mathcal{E}\left\{W\left(n^{2} N, E\right)\right\} v=0, \operatorname{logo} v^{\prime} W\left(n^{2} N, E\right) v=0$, exceto num conjunto de medida nula, isto é, quase certamente. Portanto por (2.7)

$$
\begin{aligned}
& v^{\prime}\left(A_{\theta\left(n^{2} N-1\right)}-L_{\left(n^{2} N-1\right)} C_{\theta\left(n^{2} N-1\right)}\right) \times W\left(n^{2} N-1, E, \Sigma\right) \\
& \times\left(A_{\theta\left(n^{2} N-1\right)}-L_{\left(n^{2} N-1\right)} C_{\theta\left(n^{2} N-1\right)}\right)^{\prime} v+v^{\prime} L_{\left(n^{2} N-1\right)} D_{\theta\left(n^{2} N-1\right)} \\
& \times D_{\theta\left(n^{2} N-1\right)}^{\prime} L_{\left(n^{2} N-1\right)}^{\prime} v+v^{\prime} E_{\theta\left(n^{2} N-1\right)} E_{\theta\left(n^{2} N-1\right)}^{\prime} v=0 .
\end{aligned}
$$

Como cada termo de (3.8) é não negativo e $D_{i} D_{i}^{\prime}>0$, temos que em particular para o terceiro termo:

$$
v^{\prime} L_{\left(n^{2} N-1\right)} D_{\theta\left(n^{2} N-1\right)} D_{\theta\left(n^{2} N-1\right)}^{\prime} L_{\left(n^{2} N-1\right)}^{\prime} v=0,
$$

$\operatorname{logo} L_{\left(n^{2} N-1\right)}^{\prime} v=0$, então $v^{\prime} W\left(n^{2} N, E\right) v=v^{\prime} X\left(n^{2} N, E\right) v$ e portanto

$$
v^{\prime} \mathcal{E}\left\{W\left(n^{2} N, E\right)\right\} v=v^{\prime} \mathcal{E}\left\{X\left(n^{2} N, E\right)\right\} v=0,
$$

contrariando o Lema 3.2, portanto $\mathcal{E}\left\{W\left(n^{2} N, E\right)\right\}>0$, como queríamos.

Corolário 3.5. Considere o sistema $\Phi_{M, E}$ e $W(k)$ como em (2.7). Se $(A, E, \mathbf{P})$ é w-controlável, então existe $\bar{\mu}$ tal que

$$
\mathcal{E}\left\{W\left(\ln ^{2} N\right)\right\} \geq \bar{\mu} I, \quad l \geq 1 .
$$


Demonstração: Considerando os fatos abordados no Corolário 3.3, $\mathcal{E}\left\{X\left(\ln ^{2} N\right)\right\}>$ $\mu I, \forall l \geq 1$, temos que este resultado tem prova análoga ao Corolário 3.4.

O lema abaixo faz a extensão do Corolário 3.1 para $W(k)$.

Lema 3.3. Considere o sistema $\Phi_{M, E}$ e $W$ como definido em (2.7). Se $(A, E, \mathbf{P})$ é w-controlável, então

$$
\mathcal{E}\left\{\sum_{k=1}^{l n^{2} N-1} W_{h}\left(\ln ^{2} N-1, k, \Upsilon_{k-1}(E)\right)\right\}>0, l \geq 1
$$

Demonstração: Com efeito, considere no Corolário 3.5 o caso particular em que $\Sigma=0$, tem-se por (2.9) que

$$
\mathcal{E}\left\{\sum_{k=1}^{l n^{2} N-1} W_{h}\left(\ln ^{2} N-1, k, \Upsilon_{k-1}(E)\right)\right\}=\mathcal{E}\left\{W\left(\ln ^{2} N, E, \Sigma\right)\right\}>0,
$$

como queríamos demonstrar. 


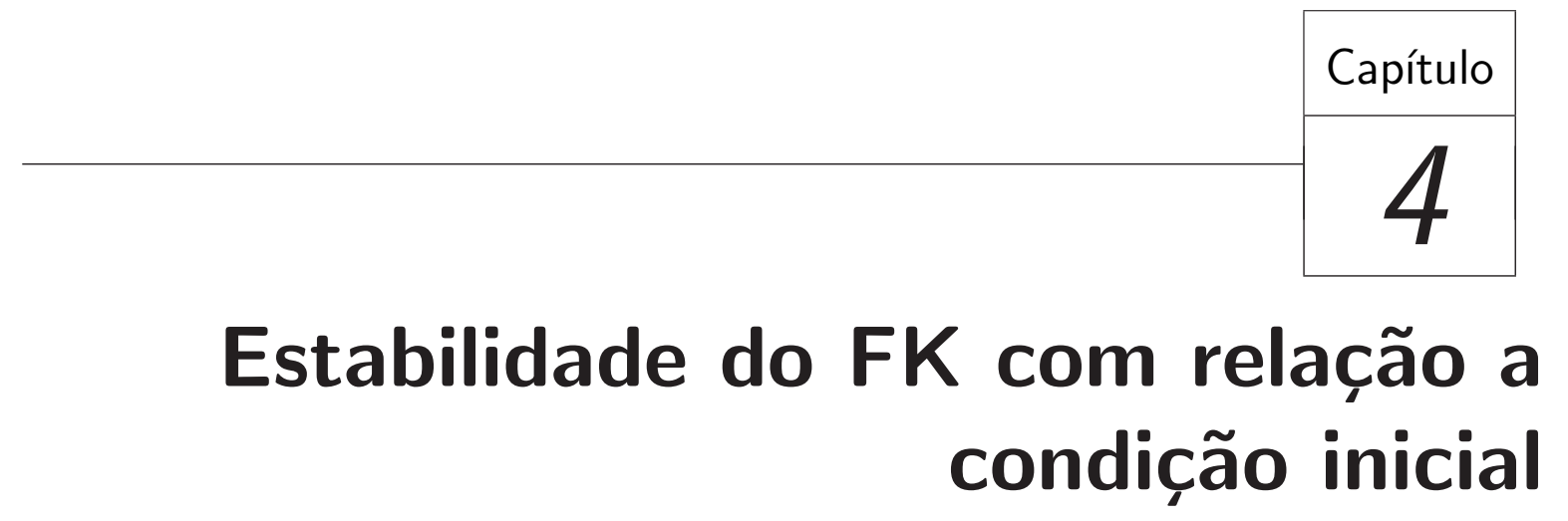

Neste capítulo apresentam-se as definições de detetabilidade estocástica e estabilidade do FK com relação à condição inicial. Esta abordagem de estabilidade já foi estudada para sistemas lineares variantes no tempo, veja [6], no sentido de que a covariância do erro de estimação seja limitada, quando há perturbações na condição inicial, i.e, $\Psi \neq \Sigma$ e quando a matriz de ruído não é perturbada, ou seja, $B=E$. Neste trabalho apresentamos esta ideia no contexto de SLSM e mostramos que controlabilidade fraca e detetabilidade estocástica são condições suficientes para a estabilidade do FK com relação a condição inicial. Exemplos numéricos ilustram os resultados.

\subsection{Estabilidade do FK em relação a $\Psi$}

Sabe-se que o FK, para sistemas lineares variantes no tempo, apresenta covariância do erro de estimação limitada quando o sistema é uniformemente detetável [2]. No cenário de sistemas lineares com saltos Markovianos, sabe-se que detetabilidade estocástica é condição suficiente para a existência de um limitante superior para o valor esperado da covariância do erro de estimação nominal do FK, veja [Theorem 1, [10]]. Noções de detetabilidade estocástica podem ser encontradas em [29] e [30].

Considera-se abaixo a noção de estabilidade para o FK e emprega-se a terminologia estabilidade com relação a condição inicial $\Psi$ (estável c.r $\Psi$ ) como em [6]. 
Definição 4.1. (Estabilidade de KF com relação a condição inicial $\Psi$ ) Dizemos que o FK é estável c.r $\Psi$ se, para cada $\Psi \in \mathcal{M}^{r 0}$, existe $\bar{P} \in \mathcal{M}^{n 0}$, tal que $\mathcal{E}\{W(k, E, \Psi)\} \leq$ $\bar{P}, k \geq 0$.

Ilustramos, no seguinte exemplo, que perturbações na condição inicial podem gerar mudanças bruscas de comportamento do FK.

Exemplo 4.1. Neste exemplo, realizamos a comparação gráfica dos comportamentos dos valores esperados da covariância do erro de estimação, para as diferentes condições iniciais tomadas abaixo. Analisamos o valor esperado da covariância do erro $\mathcal{E}\{W(k, E, \Psi)\}$ obtido através de simulação Monte Carlo com 1.000 iterações, no intervalo de tempo discreto $[0,400]$ e ilustramos na Figura 4.1 num intervalo de tempo conveniente.

Consideremos o FK para o sistema $\Phi_{M, E}$ os dados seguintes

$$
\begin{aligned}
& A_{1}=\left[\begin{array}{ccc}
0.9 & 0.1 & 0 \\
0 & 1.1 & 0 \\
0 & 0 & 0.9
\end{array}\right], A_{2}=\left[\begin{array}{ccc}
0.9 & 0.1 & 0 \\
0.1 & 0.9 & 0 \\
0 & 0 & 0.9
\end{array}\right], E_{1}=0, \quad E_{2}=\left[\begin{array}{lll}
0 & 0 & 0 \\
0 & 0 & 0 \\
0 & 0 & 1
\end{array}\right] \text {, } \\
& C_{1}=C_{2}=\left[\begin{array}{lll}
1 & 0 & 0
\end{array}\right], D_{1}=D_{2}=I_{3}, \quad \boldsymbol{P}=\left[\begin{array}{ll}
0.9 & 0.1 \\
0.9 & 0.1
\end{array}\right], \pi(0)=\left[\begin{array}{ll}
0.5 & 0.5
\end{array}\right] \text {, } \\
& \Sigma=\left[\begin{array}{ccc}
0 & 0 & 0 \\
0 & 0 & 0 \\
0.1 & 0 & 0
\end{array}\right], \tilde{\Psi}=\left[\begin{array}{ccc}
0.3 & 0 & 0 \\
0 & 0.2 & 0 \\
0 & 0 & 0.3
\end{array}\right], \bar{\Psi}=I_{3} \text { e } \hat{\Psi}=10^{-1} I_{3} .
\end{aligned}
$$

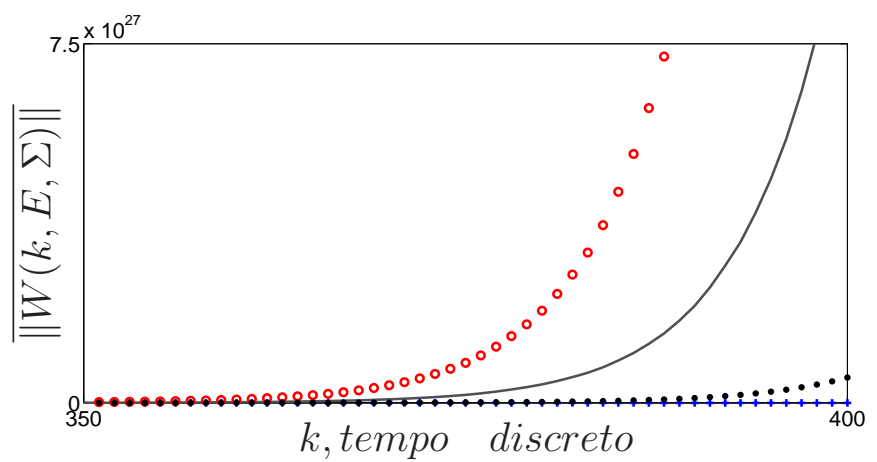

Figura 4.1: Curvas para o Exemplo 4.1: Covariância do erro de estimação

Na Figura 4.1 ilustramos $\mathcal{E}\{W(k, E, \Psi)\}$ para diferentes valores de $\Psi$, a saber $\Psi=\bar{\Psi}$, com $\mathcal{E}\{W(k, E, \Psi)\}$ representada por (o) $e \Psi=\tilde{\Psi}$ traçada como (-), nestes casos o valor esperado da covariância do erro de estimação diverge. No caso em que a condição inicial é tomada como $\Psi=\hat{\Psi}$, traçada por $(\bullet)$, apesar das curvas sugerirem 
fortemente divergência e desta forma que o FK seja instável, não temos a garantia formal (tratam-se apenas de simulações) e portanto não obtemos conclusões sobre estabilidade. Enquanto que para $\Psi=\Sigma$, com trajetória de $\mathcal{E}\{W(k, E, \Sigma)\}$ marcada por $(+)$, há convergência para $\mathcal{E}\{W(k)\}$.

O exemplo acima ilustra que neste contexto, torna-se fundamental o estudo de condições que sejam suficientes e $\backslash$ ou necessárias para limitação de $\mathcal{E}\{W(k)\}$ para quaisquer perturbações na condição inicial $\Sigma$. Mostramos na Proposição 4.1, que sob a hipótese de controlabilidade fraca e detetabilidade estocástica, existe limitante superior para $E\{W(k, E, \Psi)\}, \forall \Psi \in \mathcal{M}^{n 0}$.

Proposição 4.1. Considere $W(k, E, \Sigma)$ como definido em (2.7). Se $(A, E, \mathbf{P})$ é wcontrolável e $(A, C, \mathbf{P})$ é s-detetável, então

$$
E\{W(k, E, \Psi)\} \leq \bar{P}, \quad k \geq 0, \quad \forall \Psi \in \mathcal{M}^{n 0} .
$$

Demonstração: A prova é feita por indução matemática sobre $k$, para $k>n^{2} N$. Com efeito, para $k=n^{2} N$, pela Proposição 2.2 e pelo Corolário 3.4 existe $\bar{\mu}$ e $\epsilon \in \mathbb{R}_{+}$, $\bar{P} \in \mathcal{M}^{n 0}$ tais que,

$$
\epsilon \bar{\mu} \mathcal{E}\left\{W\left(n^{2} N, E, \Psi\right)\right\} \leq \bar{\mu} I \leq \mathcal{E}\left\{W\left(n^{2} N, E, \Sigma\right)\right\}=\mathcal{E}\left\{P\left(n^{2} N\right)\right\}<\bar{P}
$$

Para $k=n^{2} N+1,(4.1)$ implica

$$
\begin{aligned}
\mathcal{E}\left\{W\left(n^{2} N+1, \Sigma\right)\right\}- & \epsilon \bar{\mu} \mathcal{E}\left\{W\left(n^{2} N+1, \Psi\right)\right\}=\mathcal{E}\left\{W\left(n^{2} N+1, \Sigma\right)-\epsilon \bar{\mu} W\left(n^{2} N+1, \Psi\right)\right\} \\
= & \mathcal{E}\left\{\left(A_{\theta\left(n^{2} N\right)}-L_{n^{2} N} C_{\theta\left(n^{2} N\right)}\right)\left[W\left(n^{2} N, \Sigma\right)-\epsilon \bar{\mu} W\left(n^{2} N, \Psi\right)\right] \times\right. \\
& \left.\times\left(A_{\theta\left(n^{2} N\right)}-L_{n^{2} N} C_{\theta\left(n^{2} N\right)}\right)^{\prime}\right\} \geq 0,
\end{aligned}
$$

$\log 0$

$$
\epsilon \bar{\mu} \mathcal{E}\left\{W\left(n^{2} N+1, \Psi\right)\right\} \leq \mathcal{E}\left\{W\left(n^{2} N+1, \Sigma\right)\right\}=\mathcal{E}\left\{P\left(n^{2} N+1\right)\right\}<\bar{P} .
$$

Para $k=n^{2} N+\bar{k}, \bar{k} \geq 0$, assumindo que

$$
\mathcal{E}\left\{W\left(n^{2} N+\bar{k}, \Sigma\right)\right\} \geq \epsilon \bar{\mu} \mathcal{E}\left\{W\left(n^{2} N+\bar{k}, \Psi\right)\right\}
$$

então para $\bar{k}+1$ temos

$$
\begin{aligned}
& \mathcal{E}\left\{W\left(n^{2} N+\bar{k}+1\right)\right\}-\epsilon \bar{\mu} \mathcal{E}\left\{W\left(n^{2} N+\bar{k}+1, \Psi\right)\right\} \\
& =\mathcal{E}\left\{W\left(n^{2} N+\bar{k}+1\right)-\epsilon \bar{\mu} W\left(n^{2} N+\bar{k}+1, \Psi\right)\right\} \\
& =\mathcal{E}\left\{\Lambda W\left(n^{2} N+\bar{k}\right) \Lambda^{\prime}-\epsilon \bar{\mu} \Lambda_{n^{2} N+\bar{k}} W\left(n^{2} N+\bar{k}, \Psi\right) \Lambda^{\prime}\right\} \\
& =\mathcal{E}\left\{\Lambda\left[W\left(n^{2} N+\bar{k}\right)-\epsilon \bar{\mu} W\left(n^{2} N+\bar{k}, \Psi\right)\right] \Lambda^{\prime}\right\} \geq 0
\end{aligned}
$$


$\operatorname{com} \Lambda=\left(A_{\theta\left(n^{2} N+\bar{k}\right)}-C_{\theta\left(n^{2} N+\bar{k}\right)} L_{\left(n^{2} N+\bar{k}\right)}\right)$, portanto

$$
\epsilon \bar{\mu} \mathcal{E}\left\{W\left(n^{2} N+\bar{k}+1, \Psi\right)\right\} \leq \mathcal{E}\left\{W\left(n^{2} N+\bar{k}+1\right)\right\}<\bar{P},
$$

completando o processo de indução. Para $0 \leq k \leq n^{2} N-1$, o resultado é imediato do Lema 2.1.

\subsection{Exemplos Numéricos}

Na sequência apresentamos estudo de casos, variando em cada exemplo as características do sistema $\Phi_{M, E}$, tomadas como hipóteses na Proposição 4.1, a w-controlabilidade, a s-detetabilidade e a condição inicial $\Psi$. Para cada um dos casos, estudamos a estabilidade do filtro através do processo da covariância do erro $W(k)$, em termos dos valores esperados $\mathcal{E}\{\|W(k, E, \Psi)\|\}$ e $\mathcal{E}\{\|P(k)\|\}$. Para o cálculo da estimativa de $\mathcal{E}\{\|W(k, E, \Psi)\|\}$ e $\mathcal{E}\{\|P(k)\|\}$, empregamos a simulação Monte Carlo com 1.000 iterações.

Exemplo 4.2. (W-controlável e s-detetável) Considere o sitema $\Phi_{M, E}$ com

$$
\begin{gathered}
A_{1}=\left[\begin{array}{ccc}
0.9 & 0.1 & 0 \\
0 & 1.1 & 0 \\
0 & 0 & 0.9
\end{array}\right], A_{2}=\left[\begin{array}{ccc}
0.9 & 0.1 & 0 \\
0.1 & 0.9 & 0 \\
0 & 0 & 0.9
\end{array}\right], E_{1}=\left[\begin{array}{lll}
0 & 0 & 0 \\
0 & 0 & 0 \\
0 & 0 & 1
\end{array}\right], E_{2}=0, \\
C_{1}=C_{2}=\left[\begin{array}{ccc}
1 & 0 & 0
\end{array}\right], D_{1}=D_{2}=I_{3}, \pi(0)=\left[\begin{array}{cc}
0.5 & 0.5
\end{array}\right] \\
\boldsymbol{P}=\left[\begin{array}{ll}
0.1 & 0.9 \\
0.1 & 0.9
\end{array}\right], \Sigma=\left[\begin{array}{ccc}
1 & 0.3 & 0.1 \\
0.4 & 2 & 0.4 \\
0.1 & 0.3 & 1
\end{array}\right] e \Psi=\left[\begin{array}{ccc}
1.9 & 0 & 0 \\
0 & 2.7 & 0 \\
0 & 0 & 0.3
\end{array}\right] .
\end{gathered}
$$

$N a$ Figura 4.2, a curva (4.2.a) representa uma estimativa para $\mathcal{E}\{P(k)\}$ e a curva (4.2.b) representa uma estimativa para $\mathcal{E}\{W(k, E, \Psi)\}$, as quais são limitadas. Estes resultados são esperados, pela Proposição $4.1 \mathrm{w}$-controlabilidade, sob hipótese de sdetetabilidade é condição suficiente para estabilidade do FK.

Exemplo 4.3. (Não w-controlável e s-detetável) Considere o sistema $\Phi_{M, E}$, como no Exemplo 4.2, com

$$
\begin{gathered}
A_{2}=\left[\begin{array}{ccc}
0.9 & 0.1 & 0 \\
0.1 & 0.9 & 0 \\
0 & 0 & 0.9
\end{array}\right], E_{1}=0, E_{2}=\left[\begin{array}{lll}
0 & 0 & 0 \\
0 & 0 & 0 \\
0 & 0 & 1
\end{array}\right], \Sigma=\left[\begin{array}{ccc}
0 & 0 & 0 \\
0 & 0 & 0 \\
0 & 0 & 0.1
\end{array}\right] \\
e \Psi=\left[\begin{array}{ccc}
0.3 & 0 & 0 \\
0 & 0.8 & 0 \\
0 & 0 & 0.33
\end{array}\right] .
\end{gathered}
$$


$(4.2 . a)$

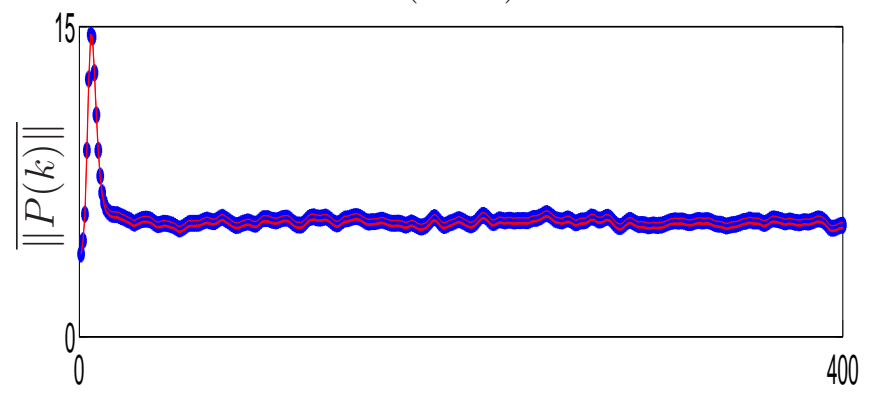

$(4.2 .6)$

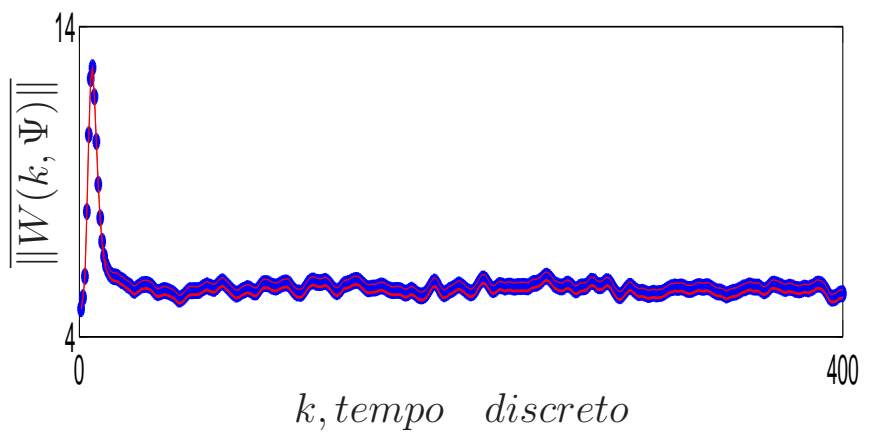

Figura 4.2: Exemplo 4.2: w-controlável e s-detetável. Estimativas para $P(k) e$ $\mathcal{E}\{W(k, \Psi)\}$ e os respectivos desvios padrões marcados com linha contínua.

Este exemplo ilustra um caso em que $(A, E, \mathbf{P})$ é não w-controlável. A Figura (4.3.a) sugere que $\mathcal{E}\{P(k)\}$ é limitada, enquanto a Figura (4.3.b) apresenta $\mathcal{E}\{W(k, E, \Psi)\}$ divergente. Portanto o KF não é estável.

Exemplo 4.4. (Não w-controlável e s-detetável) Considere o sistema $\Phi_{M, E}$, como no Exemplo 4.2, com

$$
\begin{gathered}
A_{1}=\left[\begin{array}{cc}
0.9 & 0.1 \\
0 & 1.1
\end{array}\right], A_{2}=\left[\begin{array}{cc}
0.9 & 0.1 \\
0.1 & 0.9
\end{array}\right], E_{1}=E_{2}=0, \quad C_{1}=C_{2}=\left[\begin{array}{ll}
1 & 0
\end{array}\right], \\
\boldsymbol{P}=\left[\begin{array}{ll}
0.1 & 0.9 \\
0.1 & 0.9
\end{array}\right], \pi(0)=\left[\begin{array}{ll}
0.5 & 0.5
\end{array}\right], \quad \Sigma=\left[\begin{array}{cc}
0.2 & 0.1 \\
0.1 & 0
\end{array}\right] \text { e } \Psi=\left[\begin{array}{cc}
0.5 & 0 \\
0 & 0.2
\end{array}\right] .
\end{gathered}
$$

$(A, E, \mathbf{P})$ é não w-controlável. As Figuras (4.4.a) e (4.4.b) ilustram, respectivamente, as limitações de $\mathcal{E}\{P(k)\}$ e $\mathcal{E}\{W(k, E, \Psi)\}$ e portanto o KF é estável. Este exemplo mostra que as condições apresentadas na Proposição 4.1 não são necessárias para estabilidade do FK. 
$(4.3 . a)$
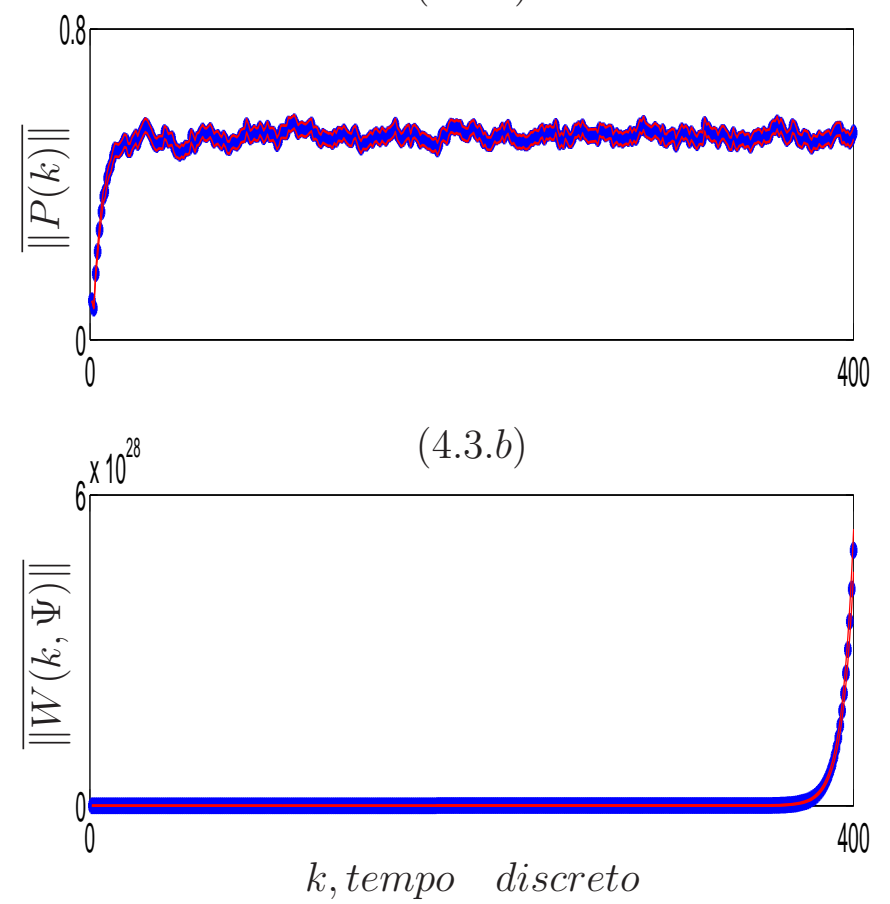

Figura 4.3: Exemplo 4.3: Não w-controlável e s-detetável. Estimativas para $P(k)$ e $\mathcal{E}\{W(k, \Psi)\}$ e os respectivos desvios padrões marcados com linha contínua.

Exemplo 4.5. (W-controlável e não s-detetável) Considere o sistema $\Phi_{M, E}$ como no Exemplo 4.2, com

$$
\begin{gathered}
A_{1}=\left[\begin{array}{ccc}
0.9 & 0 & 0.2 \\
0.1 & 1.1 & 0 \\
0.1 & 0 & 0.9
\end{array}\right], A_{2}=\left[\begin{array}{ccc}
0.9 & 0 & 0 \\
0.1 & 0.9 & 0 \\
0.1 & 0 & 0.9
\end{array}\right], E_{1}=\left[\begin{array}{lll}
1 & 1 & 0
\end{array}\right], E_{2}=\left[\begin{array}{lll}
1 & 0 & 1
\end{array}\right], \\
C_{1}=\left[\begin{array}{lll}
1.5 & 0 & 0
\end{array}\right], C_{2}=\left[\begin{array}{lll}
0.5 & 0 & 0
\end{array}\right], \boldsymbol{P}=\left[\begin{array}{cc}
0.5 & 0.5 \\
0.9 & 0.1
\end{array}\right], \pi(0)=\left[\begin{array}{ll}
0.5 & 0.5
\end{array}\right], \\
\Sigma=0 \text { e } \Psi=\left[\begin{array}{ccc}
0.9 & 0 & 0 \\
0 & 0.9 & 0 \\
0 & 0 & 0.9
\end{array}\right] .
\end{gathered}
$$

Este exemplo ilustra a hipótese de w-controlabilidade, sem a condição de s-detetabilidade, como ilustra a Figura (4.5.a), observemos na Figura (4.5.b) que o filtro de Kalman é instável. Portanto, o resultado deste caso mostra o fato de que a hipótese de sdetetabilidade é condição relevante. Sem esta condição, w-controlabilidade não é suficiente. 
$(4.4 . a)$

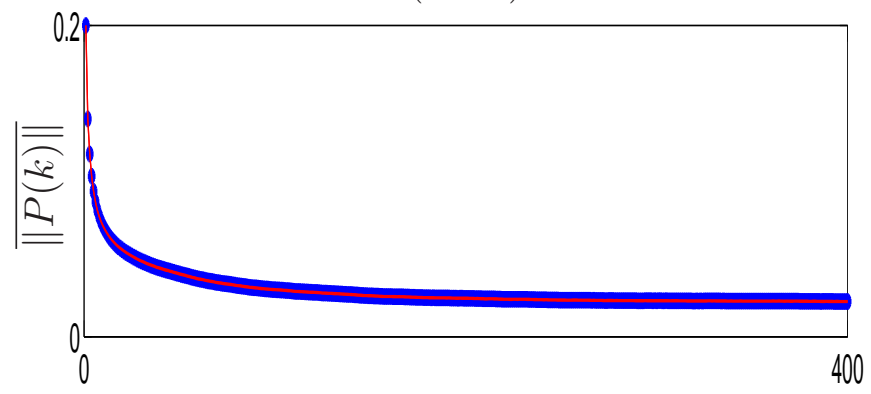

$(4.4 .6)$

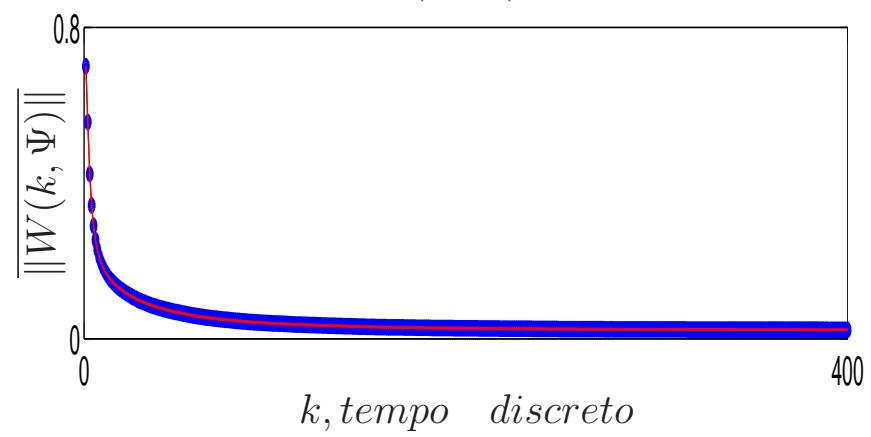

Figura 4.4: Exemplo 4.4: Não w-controlável e s-detetável. Estimativas para $P(k)$ e $\mathcal{E}\{W(k, \Psi)\}$ e os respectivos desvios padrões marcados com linha contínua.

Resumidamente os exemplos apresentados ilustram a validade do resultado obtido na Proposição 4.1, sendo que o Exemplo 4.4 mostra que as condições não são necessárias. Na Tabela 4.2 resumimos os resultados obtidos nos exemplos. ${ }^{1}$

Tabela 4.1: Tabela resumo: Estabilidade c.r $\Psi$

\begin{tabular}{|c|c|c|c|}
\hline Caso & w-controlabilidade & s-detetabilidade & estabilidade do FK \\
\hline \hline Exemplo 4.2 & $\checkmark$ & $\checkmark$ & $\checkmark$ \\
\hline Exemplo 4.3 & & $\checkmark$ & \\
\hline Exemplo 4.4 & & $\checkmark$ & $\checkmark$ \\
\hline Exemplo 4.5 & $\checkmark$ & & \\
\hline
\end{tabular}

\footnotetext{
1 Obs: $\checkmark$ indica "sim".
} 

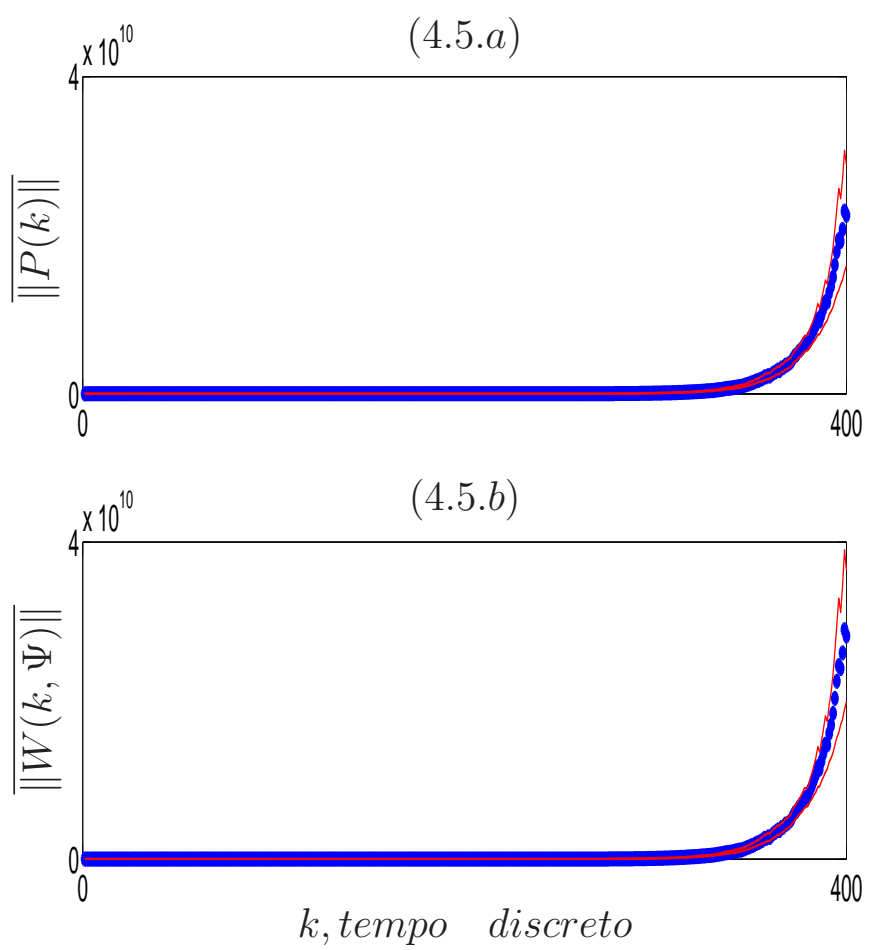

Figura 4.5: Exemplo 4.5: w-controlável e não s-detetável. Estimativas para $P(k)$ e $\mathcal{E}\{W(k, \Psi)\}$ e os respectivos desvios padrões marcados com linha contínua. 


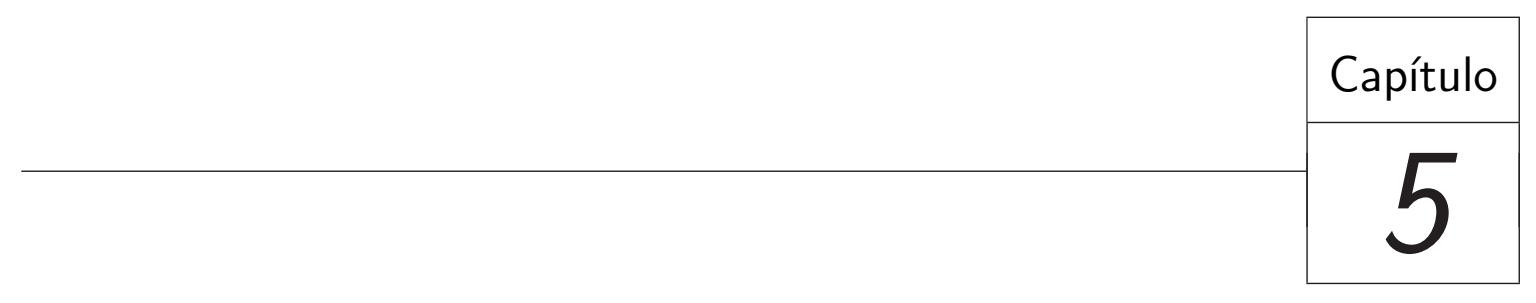

\section{Estabilidade do Filtro de Kalman}

Tratamos nos capítulos anteriores a estabilidade com respeito a condição inicial $\Psi$. É de grande interesse teórico e prático abordar a estabilidade considerando erro no termo que introduz ruído $E$, além da condição inicial $\Sigma$. Para o caso de sistemas lineares invariantes no tempo, esta questão possui um estudo bem abrangente, com a inclusão de condições necessárias e suficientes envolvendo estabilidade do FK, veja [7] e [11].

Neste trabalho, considera-se o conceito de estabilidade do FK como na definição de estabilidade adotada por [6], [7], [11], [32], [37] e [40]. Mostra-se que controlabilidade fraca e detetabilidade estocástica são condições suficientes para a estabilidade. Apresentamos resultados parciais referentes a um estudo em andamento, contudo sob uma hipótese adicional na cadeia de Markov, além de uma conjectura. Exemplos ilustrativos são incluídos. Assumimos que o FK seja calculado levando em conta que os ruídos gaussianos tenham matrizes de covariância descritas por $E_{i}$ e $\Sigma$, quando na verdade as covariância reais são $B_{i}$ e $\Psi$ respectivamente.

\subsection{Abordagem usual de estabilidade do FK}

Busca-se a existência de $\tilde{P} \in \mathcal{M}^{n 0}$ tal que o valor esperado da covariância do erro de estimação seja limitado, i.e, $\mathcal{E}\{W(k, B, \Psi)\}<\tilde{P}, k \geq 0$. Dizemos que o FK satisfazendo estas condições é estável. Definimos estabilidade do FK de forma usual, como em [6]. 
Definição 5.1. (Estabilidade do FK) Diz-se que o FK é estável se, para cada $B \in \mathcal{M}^{n, p}$ e $\Psi \in \mathcal{M}^{n 0}$, existe $\tilde{P} \in \mathcal{M}^{n 0}$ tal que $\mathcal{E}\{W(k, B, \Psi)\}<\tilde{P}, \quad k \geq 0$.

Para os resultados obtidos nesta seção, assumimos que a cadeia de Markov seja equilibrada, como na suposição abaixo.

Suposição 1. Assumimos que a distribuição inicial da cadeia de Markov é equilibrada, i.e, $\pi_{0} \mathbf{P}=\pi_{0}$.

Nesta abordagem terá um papel importante a seguinte variável aleatória

$$
W\left((l+1) n^{2} N, 0, E, \Sigma\right), \quad l, n, N \in \mathbb{N},
$$

que representa a covariância do erro de estimação no intervalo de tempo $(0,(l+$ 1) $\left.n^{2} N\right), \quad l \geq 0$, com condição inicial $\Sigma$, definida recursivamente via

$$
\left\{\begin{aligned}
W(m, r, E, \Sigma) & =\Lambda_{m-1} W(m-1, r, E, \Sigma) \Lambda_{m-1}^{\prime}+L_{m-1} D_{m-1} D_{m-1}^{\prime} L_{m-1}^{\prime} \\
& +E_{m-1} E_{m-1}^{\prime}, \quad m \geq r \\
W(r, r, E, \Sigma) & =\Sigma
\end{aligned}\right.
$$

com $\Lambda_{k}=A_{\theta(k)}-L_{k} C_{\theta(k)}, \quad D_{i}, E_{i} \in \mathcal{M}^{n, r}, i \in \mathbb{S}$ e $L_{k}$ o ganho de Kalman como definido em (2.5). A solução de (5.1) pode ser escrita como

$$
W\left(m, r, E, \Sigma_{r}\right)=W(m, r, E=0, W(r, 0, E=0, \Sigma))+\sum_{k=r+1}^{m-1} W_{h}\left(m-1, k, \Upsilon_{k-1}(E)\right)
$$

onde $W_{h}$ é como definido em (2.8)

$$
W_{h}(m, r, V)=\Lambda_{m} \Lambda_{m-1} \ldots \Lambda_{r} V \Lambda_{r}^{\prime} \ldots \Lambda_{m-1}^{\prime} \Lambda_{m}^{\prime}, \quad V \in \mathcal{M}^{n, r}
$$

e

$$
\Upsilon_{k}(E)=L_{k} D_{\theta(k)} D_{\theta(k)}^{\prime} L_{k}^{\prime}+E_{\theta(k)} E_{\theta(k)}^{\prime}
$$

Por facilidade de notação, introduzimos a variável aleatória

$$
\Sigma_{l}=W\left(\ln ^{2} N, E, \Sigma\right)
$$

sendo $\Sigma$ o valor disponível para $\Psi$, lembrando que em geral $\Sigma \neq \Psi$. Com o que podemos escrever

$$
\begin{aligned}
W\left((l+1) n^{2} N, 0, E, \Sigma\right) & =W\left((l+1) n^{2} N, E=0, \Sigma\right) \\
& +\sum_{k=1}^{(l+1) n^{2} N-1} W_{h}\left((l+1) n^{2} N-1, k, \Upsilon_{k-1}(E)\right)
\end{aligned}
$$




$$
\begin{aligned}
& =\tilde{X}\left((l+1) n^{2} N, \ln ^{2} N, E=0, \Sigma_{l}\right) \\
& +\sum_{k=l n^{2} N+1}^{(l+1) n^{2} N-1} W_{h}\left((l+1) n^{2} N-1, k, \Upsilon_{k-1}(E)\right) \\
& =W\left((l+1) n^{2} N, \ln ^{2} N, E, \Sigma_{l}\right),
\end{aligned}
$$

sendo $W\left((l+1) n^{2} N, \ln ^{2} N, E, \Sigma\right)$, a covariância do erro de estimação no instante $(l+$ 1) $n^{2} N, l \geq 1$ e "condição inicial" $\Sigma_{l}$.

Observação 4. Observemos que existe $\bar{\Lambda} \in \mathcal{M}^{n 0}$, tal que $\mathcal{E}\left\{\left\|A_{\theta(k)}-L_{k} C_{\theta(k)}\right\|\right\}<\bar{\Lambda}$. De fato, obtém-se pelo Lema 2.1 que $\mathcal{E}\left\{\left\|L_{k}\right\|\right\} \leq \bar{L}$, logo $\mathcal{E}\left\{\left\|A_{\theta(k)}-L_{k} C_{\theta(k)}\right\|\right\} \leq$ $\|A\|+E\left\{\left\|L_{k}\right\|\right\}\|C\| \leq \bar{\Lambda}$.

Com o objetivo de obter um limitante superior $\tilde{P}$ de $W(k, 0, B, \Psi)$ para quaisquer $B_{i} \in \mathcal{M}^{r, n}$ e $\Psi \in \mathcal{M}^{n 0}$, seguiremos as etapas abaixo.

1. Buscamos um escalar positivo $\varepsilon$ e $\Xi \in \mathcal{M}^{n 0}$, tais que seja construída uma "trajetória auxiliar" $W(k, \varepsilon I, \Xi)$, a qual em valor esperado é limitante inferior de $\mathcal{E}\{W(k, 0, E, \Sigma)\}$ nos pontos da forma $k=\ln ^{2} N, l \geq 1$. Mais especificamente, toma-se $\Xi_{l} \leq \Sigma_{l}$ e busca-se uma desigualdade da seguinte forma

$$
\mathcal{E}\left\{W\left((l+1) n^{2} N, \ln ^{2} N, \varepsilon I, \Xi_{l}\right)\right\} \leq \mathcal{E}\left\{W\left((l+1) n^{2} N, \ln ^{2} N, E, \Sigma_{l}\right)\right\} .
$$

Inicialmente considera-se $l=0$, para determinação do valor de $\varepsilon$ e em seguida estende-se a desigualdade em (5.4) para $l \geq 1$, de forma a manter a uniformidade de $\varepsilon$ para os valores não triviais de $l$; nesta perspectiva, quando para variáveis aleatórias $U \in \mathcal{M}^{r, n}$ e $V \in \mathcal{M}^{n 0}$, é valida a seguinte desigualdade

$$
\mathcal{E}\left\{W\left((l+1) n^{2} N, \ln ^{2} N, U, V\right)\right\}=\mathcal{E}\left\{W\left((l+1) n^{2} N, \ln ^{2} N, U, \mathcal{E}\{V\}\right)\right\},
$$

conclui-se que

$$
\mathcal{E}\left\{W\left(\ln ^{2} N, 0, \varepsilon I, \Xi\right)\right\} \leq \mathcal{E}\left\{W\left(\ln ^{2} N, 0, E, \Sigma\right)\right\} \leq \bar{P}, \forall l \geq 1, \quad \bar{P} \in \mathcal{M}^{n 0}
$$

na qual, a desigualdade a direita é proveniente de [10, Theorem 1]. Veja o Corolário 5.1.

2. Empregando algumas propriedades usuais do valor esperado e de matrizes e o resultado anterior, obtemos pela w-controlabilidade de $(A, \varepsilon I, P)$, um escalar positivo $\delta$ que satisfaça a seguinte desigualdade

$$
\mathcal{E}\left\{W\left(\ln ^{2} N, \delta B, \delta \Psi\right)\right\} \leq \mathcal{E}\left\{W\left(\ln ^{2} N, \varepsilon I, \Xi\right)\right\} \leq \bar{P} .
$$


Com esta finalidade, ajusta-se $\delta$ tal que

$$
\mathcal{E}\left\{\sum_{k=1}^{l n^{2} N-1} W\left(\ln ^{2} N-1, k, \Upsilon_{k-1}(\delta B)\right)\right\}<\mathcal{E}\left\{\sum_{k=1}^{l n^{2} N-1} W\left(\ln ^{2} N-1, k, \Upsilon_{k-1}(\varepsilon I)\right)\right\}
$$

e $\delta \Psi \leq \Xi$. Vide Lema 5.1 .

3. Finalmente, elaboramos algumas avaliações mostrando que existe $\tilde{P} \in \mathcal{M}^{n 0}$ tal que (5.6) vale para todo instante $k \geq 0$, ou seja

$$
\mathcal{E}\{W(k, 0, B, \Psi)\} \leq \tilde{P}
$$

Levando a estabilidade do FK, veja o Teorema 2.

Na sequência desenvolvemos as etapas acima. O Teorema 1 fornece, sob certa hipótese, $\varepsilon \in \mathbb{R}_{+}$e $U \in \mathcal{M}^{n 0}$, tal que seja válida uma expressão similar a (5.4), para um intervalo qualquer de tempo discreto $(m+1, r), m, r \in \mathbb{N}$. Em seguida, mostra-se no Corolário 5.1 que o próximo resultado é válido, em particular, para intervalos da forma $\left(\ln ^{2} N,(l+\right.$ 1) $\left.n^{2} N\right)$ sob hipótese de w-controlabilidade.

Teorema 1. Considere $W(k)$, como definido em (5.2). Se

$$
\mathcal{E}\left\{\sum_{k=r+1}^{m-1} W_{h}\left(m-1, k, \Upsilon_{k-1}(E)\right)\right\}>\rho I, \rho \in \mathbb{R}_{+}, m, r \in \mathbb{N}: m \geq r
$$

então existe $\varepsilon \in \mathbb{R}_{+}$dependendo de $\rho$ e de $m-r$, tal que

$$
\mathcal{E}\{W(m+1, r, \varepsilon I, U)\} \leq \mathcal{E}\{W(m+1, r, E, V)\}, \quad U, V \in \mathcal{M}^{n 0}
$$

sempre que $U \leq V$.

Demonstração: Com efeito, tomando $U \leq V$, diretamente pela solução da covariância do erro de estimação $W(k)$ em (5.2) obtém-se

$$
\begin{aligned}
& W(m, r, E=0, U)=W_{h}(k, r, U)=\Lambda_{m} \Lambda_{m-1} \ldots \Lambda_{r} U \Lambda_{r}^{\prime} \ldots \Lambda_{m-1}^{\prime} \Lambda_{m}^{\prime} \\
& \leq \Lambda_{m} \Lambda_{m-1} \ldots \Lambda_{r} V \Lambda_{r}^{\prime} \ldots \Lambda_{m-1}^{\prime} \Lambda_{m}^{\prime}=W_{h}(k, r, V)=W(m, r, E=0, V)
\end{aligned}
$$

levando a

$$
\mathcal{E}\{W(m, r, E=0, U)\} \leq \mathcal{E}\{W(m, r, E=0, V)\}
$$

e existe $\varepsilon \in \mathbb{R}_{+}$, pequeno o suficiente, tal que

$$
\mathcal{E}\left\{\sum_{k=r+1}^{m-1} W_{h}\left(m-1, k, E_{\theta(k-1)} E_{\theta(k-1)}^{\prime}\right)\right\} \geq \mathcal{E}\left\{\sum_{k=r+1}^{m-1} W_{h}\left(m-1, k, \varepsilon^{2} I\right)\right\} .
$$


Além disso, desenvolvendo o segundo termo de (5.2), obtemos

$$
\begin{aligned}
\mathcal{E}\left\{\sum_{k=r+1}^{m-1} W_{h}\left(m-1, k, \Upsilon(E)_{k-1}\right)\right\} & =\mathcal{E}\left\{\sum_{k=r+1}^{m-1} W_{h}\left(m-1, k, L_{k-1} D_{\theta(k-1)} D_{\theta(k-1)}^{\prime} L_{k-1}\right)\right\} \\
& +\mathcal{E}\left\{\sum_{k=r+1}^{m-1} W_{h}\left(m-1, k, E_{\theta(k-1)} E_{\theta(k-1)}^{\prime}\right)\right\},
\end{aligned}
$$

logo por (5.10) e empregando (5.11) tem-se

$$
\mathcal{E}\left\{\sum_{k=r+1}^{m-1} W_{h}\left(m-1, k, \Upsilon_{k-1}(E)\right)\right\} \leq \mathcal{E}\left\{\sum_{k=r+1}^{m-1} W_{h}\left(m-1, k, \Upsilon_{k-1}(\varepsilon I)\right)\right\}
$$

portanto, através de (5.9) e (5.12) empregando (5.2) avalia-se

$$
\begin{aligned}
\mathcal{E}\{W(m, r, \varepsilon I, U)\} & =\mathcal{E}\{W(m, r, 0, U)\}+\mathcal{E}\left\{\sum_{k=r+1}^{m-1} W_{h}\left(m-1, k, \Upsilon_{k-1}(\varepsilon I)\right)\right\} \\
& \leq \mathcal{E}\{W(m, r, 0, V)\}+\mathcal{E}\left\{\sum_{k=r+1}^{m-1} W_{h}\left(m-1, k, \Upsilon_{k-1}(E)\right)\right\} \\
& =\mathcal{E}\{W(m, r, E, V)\}
\end{aligned}
$$

concluindo a demonstração. Sendo importante ressaltar que dada a hipótese considerada na Suposição 1, pode-se mostrar que o valor de $\varepsilon$ em (5.10) depende dos valores de $m$ e $r$ apenas, no que se refere a sua diferença, basta comparar o valor de $\rho$ com o tamanho do intervalo $[m, r]$.

O corolário a seguir trata da obtenção de um $\varepsilon>0$ que satisfaça (5.8) para as informações do sistema nominal $E$ e $\Sigma$ no intervalo de tempo $\left[\ln ^{2} N,(l+1) n^{2} N\right]$, sob a condição de controlabilidade fraca e detetabilidade estocástica. Assumimos a seguinte conjectura como verdadeira.

Conjectura 1. Considere $W(k)$ como definido em (5.3), $U \in \mathcal{M}^{r, n}$ e $V \in \mathcal{M}^{n 0}$ variáveis aleatórias, então

$$
\mathcal{E}\left\{W\left((l+1) n^{2} N, \ln ^{2} N, U, V\right)\right\}=\mathcal{E}\left\{W\left((l+1) n^{2} N, \ln ^{2} N, U, \mathcal{E}\{V\}\right)\right\}
$$

Consideramos a seguir, $\Xi_{l}=W\left(\ln ^{2} N, \varepsilon I, \Xi\right)$, sendo $\Xi \in \mathcal{M}^{n 0}$.

Corolário 5.1. Considere a covariância do erro de estimação $W(k)$, definida em (5.2). Se $(A, E, \mathbf{P})$ é w-controlável, então existe $\varepsilon>0$ tal que, para $\Xi \leq \Sigma$, tem-se

$$
\mathcal{E}\left\{W\left((l+1) n^{2} N, 0, \varepsilon I, \Xi\right)\right\}<\mathcal{E}\left\{W\left((l+1) n^{2} N, 0, E, \Sigma\right)\right\}, \quad \forall l \geq 1 .
$$


Demonstração: Temos pelo Lema 3.3 que w-controlabilidade fornece

$$
\mathcal{E}\left\{\sum_{k=1}^{l n^{2} N-1} W_{h}\left(\ln ^{2} N-1, k, \Upsilon_{k-1}(E)\right)\right\}>\bar{\rho} I, \quad \forall l \geq 1, \quad \bar{\rho} \in \mathbb{R}_{+},
$$

Por outro lado, definindo $\bar{\Sigma}_{l}=\mathcal{E}\left\{W\left(\ln ^{2} N, E, \Sigma_{l-1}\right)\right\}, \bar{\Xi}_{l}=\mathcal{E}\left\{W\left(\ln ^{2} N, E, \Xi_{l-1}\right)\right\}, l \geq$ 1 e tomando $\bar{\Xi}=\Xi \leq \Sigma=\bar{\Sigma}$, mostra-se indutivamente que $\bar{\Xi}_{l} \leq \bar{\Sigma}_{l}, \quad \forall l \geq 0$. De fato, por definição, temos $\bar{\Xi} \leq \bar{\Sigma}$; assumindo que a hipótese vale para $l>0$, isto é, $\bar{\Xi}_{l} \leq \bar{\Sigma}_{l}$, de (5.15) e pelo Teorema 1 obtém-se $\varepsilon \in \mathbb{R}_{+}$tal que

$$
\mathcal{E}\left\{W\left((l+1) n^{2} N, \ln ^{2} N, \varepsilon I, \bar{\Xi}_{l}\right)\right\} \leq \mathcal{E}\left\{W\left((l+1) n^{2} N, \ln n^{2} N, \varepsilon I, \bar{\Sigma}_{l}\right)\right\}
$$

e considerando a Conjectura 1, obtemos

$$
\begin{aligned}
& \mathcal{E}\left\{W\left(\ln ^{2} N, 0, \varepsilon I, \Xi_{0}\right)\right\}=\mathcal{E}\left\{W\left(\ln ^{2} N,(l-1) n^{2} N, \varepsilon I, \Xi_{l-1}\right)\right\} \\
& =\mathcal{E}\left\{W\left(\ln ^{2} N,(l-1) n^{2} N, \varepsilon I, \mathcal{E}\left\{\Xi_{l-1}\right\}\right)\right\}=\mathcal{E}\left\{W\left(\ln ^{2} N,(l-1) n^{2} N, \varepsilon I, \bar{\Xi}_{l-1}\right)\right\} \\
& \leq \mathcal{E}\left\{W\left(\ln ^{2} N,(l-1) n^{2} N, \varepsilon I, \bar{\Sigma}_{l-1}\right)\right\}=\mathcal{E}\left\{W\left(\ln ^{2} N,(l-1) n^{2} N, E, \mathcal{E}\left\{\Sigma_{l-1}\right\}\right)\right\} \\
& =\mathcal{E}\left\{W\left(\ln ^{2} N,(l-1) n^{2} N, E, \Sigma_{l-1}\right)\right\}=\mathcal{E}\left\{W\left(\ln ^{2} N, 0, E, \Sigma_{0}\right)\right\}
\end{aligned}
$$

Concluindo a demonstração. Lembrando que o valor de $\varepsilon$ depende apenas de $n^{2} N$, com efeito, $\left(l+1 n^{2} N-l n^{2} N\right)=n^{2} N$, portanto a uniformidade de $\varepsilon$ como tomada em (5.16) é verdadeira.

Para os resultados seguintes, é importante que a trajetória percorrida pelo valor esperado da covariância do erro de estimação $\mathcal{E}\left\{W\left(\ln ^{2} N, \varepsilon I, \Xi_{0}\right)\right\}$ seja positiva, este fato é proveniente da w-controlabilidade de $(A, \varepsilon I, P)$. De fato, pelo Corolário 3.5, existe um escalar positivo $\mu_{\varepsilon}>0$ tal que $\mathcal{E}\left\{W\left(\ln ^{2} N, \varepsilon I, \Xi_{l-1}\right)\right\} \geq \mu_{\varepsilon}$, portanto a trajetória auxiliar $\mathcal{E}\left\{W\left(k, \varepsilon I, \Xi_{0}\right)\right\}$ possui limitante inferior positivo para os pontos da forma $k=\ln ^{2} N, l \geq 1$.

Lema 5.1. Considere $W(k)$, como definido em (2.7). Se $(A, E, \mathbf{P})$ é w-controlável e $(A, C, \mathbf{P})$ é s-detetável, então existe $\delta \in \mathbb{R}_{+}$e $\bar{P} \in \mathcal{M}^{n 0}$ tal que

$$
\mathcal{E}\left\{W\left(\ln ^{2} N, \delta B, \delta \Psi\right)\right\}<\bar{P}, l \geq 0 .
$$

Demonstração: Como $(A, \varepsilon I, \mathbf{P})$ é w-controlável, então pelo Lema 3.3, tem-se

$$
\mathcal{E}\left\{\sum_{k=1}^{l n^{2} N-1} W_{h}\left(\ln ^{2} N-1, k, \Upsilon_{k-1}(\varepsilon I)\right)\right\}>\rho_{\varepsilon} I, \rho_{\varepsilon} \in \mathbb{R}_{+},
$$


e pode-se tomar $\delta$, pequeno o suficiente, tal que

$$
\begin{aligned}
& \mathcal{E}\left\{\sum_{k=1}^{\ln n^{N}-1} W\left(\ln ^{2} N-1, k, \Upsilon_{k-1}(\delta B)\right)\right\} \leq \mathcal{E}\left\{\sum_{k=1}^{l n^{2} N-1} W\left(\ln ^{2} N-1, k, \Upsilon_{k-1}(\varepsilon I)\right)\right\} \\
& e \delta \Psi \leq \Xi .
\end{aligned}
$$

Portanto, empregando (5.2) através de (5.17) e considerando a ideia na obtenção de (5.9), podemos escrever

$$
\begin{aligned}
\mathcal{E}\left\{W\left(\ln ^{2} N, \delta B, \delta \Psi\right)\right\} & =\mathcal{E}\left\{W\left(\ln ^{2} N, 0, \delta \Psi\right)\right\}+\mathcal{E}\left\{\sum_{k=1}^{\ln 2} W\left(\ln ^{2} N-1, k, \Upsilon_{k-1}(\delta B)\right)\right\} \\
& \leq \mathcal{E}\left\{W\left(\ln ^{2} N, 0, \Xi\right)\right\}+\mathcal{E}\left\{\sum_{k=1}^{l n^{2} N-1} W\left(\ln ^{2} N-1, k, \Upsilon_{k-1}(\varepsilon I)\right)\right\} \\
& =\mathcal{E}\left\{W\left(\ln ^{2} N, \varepsilon I, \Xi\right)\right\} \leq \bar{P},
\end{aligned}
$$

com o que concluímos

$$
\left.\mathcal{E}\left\{W\left(\ln ^{2} N, B, \Psi\right)\right\}\right\} \leq \delta^{-1} \bar{P}
$$

como desejado.

Obtemos condições de limitação para o valor esperado da covariância do erro de estimação $W(k, B, \Psi)$ nos pontos $k=l n^{2} N$, para obter resultado de estabilidade mostra-se que a limitação pode ser estendida a todos os pontos $k \geq 0$. Obtém-se que o crescimento de $\mathcal{E}\{W(k)\}$ é sempre limitado em cada intervalo da forma $\left[\ln ^{2} N,(l+1) n^{2} N\right]$. Como há uma limitação $\hat{M}=\delta^{-1} \bar{P} \in \mathcal{M}^{n 0}$ fixa para o valor esperado de $W\left(\ln ^{2} N, B, \Psi\right)$, para todo $l \geq 0$, tomamos $\mathcal{E}\left\{W\left(\ln ^{2} N, B, \Psi_{l-1}\right)\right\} \leq \hat{M}, l \geq 1$ e segue o resultando de estabilidade do FK.

Teorema 2. Se $(A, E, \mathbf{P})$ é w-controlável e $(A, C, \mathbf{P})$ é s-detetável, então o $F K$ é estável.

Demonstração: Mostramos que para $k \in\left[\ln ^{2} N,(l+1) n^{2} N\right], \forall l \geq 0$, existe um limitante máximo para $\mathcal{E}\{W(k, B, \Psi)\}$. Com efeito, considere $\bar{\Psi}_{l}=\mathcal{E}\left\{W\left(\ln ^{2} N, B, \Psi_{l-1}\right)\right\}$ e $\bar{k} \in\left[\ln ^{2} N,(l+1) n^{2} N\right]$, tem-se

$$
\begin{aligned}
\mathcal{E}\{W(\bar{k}, 0, B, \Psi)\} & =\mathcal{E}\left\{W\left(\bar{k}, \ln ^{2} N, B, \bar{\Psi}_{l}\right)\right\} \\
& =\mathcal{E}\left\{W\left(\bar{k}, \ln ^{2} N, 0, \bar{\Psi}_{l}\right)\right\}+\mathcal{E}\left\{\sum_{k=l n^{2} N+1}^{\bar{k}-1} W_{h}\left(\bar{k}-1, k, \Upsilon_{k-1}(B)\right)\right\} \\
& \leq\|\Lambda\|^{\bar{k}}\left\|\bar{\Psi}_{l}\right\| I+\bar{k}\|\Upsilon\|\|\Lambda\|^{k-1} I \\
& \leq\|\Lambda\|^{\bar{k}} \hat{M}+\bar{k}\|\Upsilon\|\|\Lambda\|^{k-1}=\tilde{P}
\end{aligned}
$$


$\operatorname{com}\|\Lambda\|=\max _{\theta(k) \in \mathbb{S}}\left\{\mathcal{E}\left\{A_{\theta(k)}-L_{k} C_{\theta(k)}\right\}\right\}$,

$\|\Upsilon\|=\max _{\theta(k) \in \mathbb{S}}\left\{\mathcal{E}\left\{\left\{k D_{\theta(k)} D_{\theta(k)}^{\prime} L_{k}+\right\} \theta(k) B_{\theta(k)}^{\prime}\right\}\right\}$ e $0 \leq k \leq \bar{k}$. Portanto o FK é estável.

O Teorema 2 fornece um resultado de estabilidade do FK, fornecendo condições suficientes para estabilidade. Uma questão que surge naturalmente é a busca por condições que sejam necessárias e suficientes para estabilidade para cadeias de Markov mais gerais e sem a adição da Conjectura 1.

\subsection{Exemplos Numéricos}

Na sequência apresentamos casos de estudo, variando as características do sistema $\Phi_{M, E}$, tomadas como hipóteses no Teorema 2, a condição de w-controlabilidade, sdetetabilidade, a condição inicial $\Psi$ e o termo de ruído $B_{i}, i \in \mathbb{S}$. Para cada um dos casos, analisamos a estabilidade do filtro através do processo da covariância do erro $W(k)$ em termo dos valores esperados $\mathcal{E}\{W(k, B, \Psi)\}$ e $\mathcal{\varepsilon}\{P(k)\}$.

Para o cálculo da estimativa de $\mathcal{E}\{W(k, B, \Psi)\}$ e $\mathcal{E}\{P(k)\}$, empregamos a simulação Monte Carlo com 1.000 iterações.

Exemplo 5.1. (W-controlável e s-detetável) Considere o sistema $\Phi_{M}$ com

$$
\begin{aligned}
& A_{1}=\left[\begin{array}{ccc}
0.3 & 0.2 & 0 \\
0 & 1.1 & 0 \\
0.6 & 0 & 0.9
\end{array}\right], A_{2}=\left[\begin{array}{lll}
0.1 & 0.2 & 0 \\
0.3 & 0.1 & 0 \\
0.2 & 0.1 & 0
\end{array}\right], E_{1}=\left[\begin{array}{ccc}
0 & 0 & 0 \\
0 & 0.1 & 0 \\
0.1 & 0 & 0
\end{array}\right], \\
& E_{2}=\left[\begin{array}{ccc}
0 & 0 & 0 \\
0 & 0.1 & 0 \\
0 & 0 & 0
\end{array}\right], B_{1}=\left[\begin{array}{ccc}
0.1 & 0 & 0 \\
0 & 0.9 & 0 \\
0 & 0 & 0.11
\end{array}\right], B_{2}=\left[\begin{array}{ccc}
0.1 & 0 & 0 \\
0 & 0.3 & 0 \\
0 & 0 & 0.1
\end{array}\right] \\
& C_{1}=C_{2}=\left[\begin{array}{lll}
1 & 0 & 0
\end{array}\right], D_{1}=D_{2}=I_{3}, \quad \boldsymbol{P}=\left[\begin{array}{cc}
0.5 & 0.5 \\
0.1 & 0.9
\end{array}\right], \\
& \pi(0)=\left[\begin{array}{ll}
0.5 & 0.5
\end{array}\right], \Sigma=0 \text { e } \Psi=\left[\begin{array}{ccc}
0.3 & 0 & 0 \\
0 & 0.2 & 0 \\
0 & 0 & 0.3
\end{array}\right]
\end{aligned}
$$

Pelo teste de controlabilidade, obtemos que $(A, E, \mathbf{P})$ é w-controlável, como $(A, C, \mathbf{P})$ foi tomada s-detetável, pelo resultado do Teorema 2, o FK é estável. Para ilustração, na Figura (5.1.a) verifica-se a limitação de $\mathcal{E}\{P(k)\}$ garantida pela hipótese de sdetetabilidade, enquanto na Figura (5.1.b) verifica-se a limitação de $\mathcal{E}\{W(k, B, \Psi)\}$. 
$(5.1 . a)$

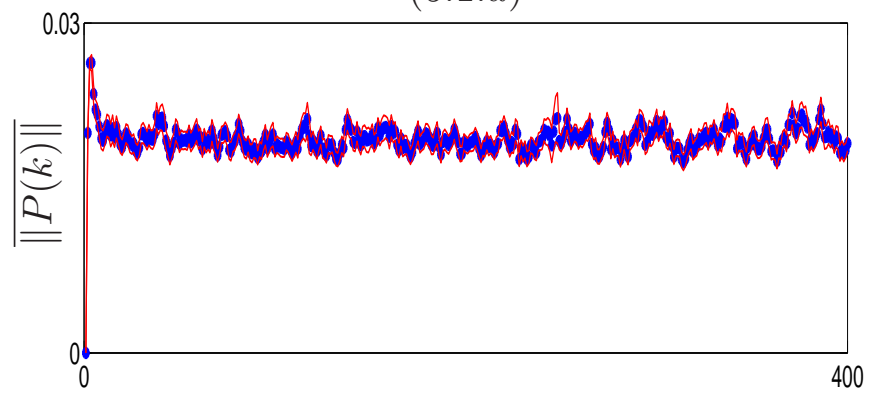

$(5.1 .6)$

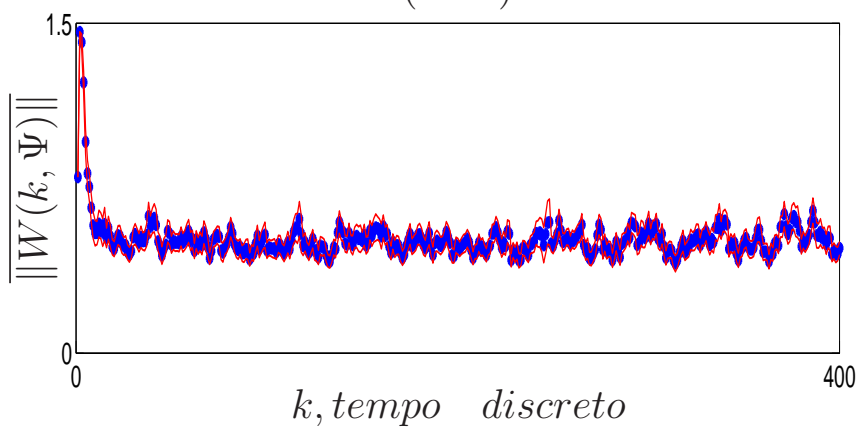

Figura 5.1: Exemplo 5.1: w-controlável e s-detetável. Estimativas para $P(k)$ e $\mathcal{E}\{W(k, B, \Psi)\}$ e os respectivos desvios padrões marcados com linha contínua.

Exemplo 5.2. ( Não w-controlável e s-detetável) Considere o sistema $\Phi_{M}$, como no Exemplo $5.1 \mathrm{com}$

$$
\begin{gathered}
A_{1}=\left[\begin{array}{ccc}
0.9 & 0.1 & 0 \\
0 & 1.1 & 0 \\
0 & 0 & 0
\end{array}\right], A_{2}=\left[\begin{array}{lll}
0.9 & 0.4 & 0 \\
0.1 & 0.9 & 0 \\
0.1 & 0.5 & 0.9
\end{array}\right], E_{1}=0, E_{2}=\left[\begin{array}{lll}
0 & 0 & 0 \\
0 & 0 & 0 \\
0 & 0 & 1
\end{array}\right], \\
B_{1}=0.1^{100} I_{3}, \quad B_{2}=\left[\begin{array}{ccc}
0.1 & 0 & 0 \\
0 & 0.4 & 0 \\
0 & 0 & 1
\end{array}\right], C_{1}=C_{2}=\left[\begin{array}{lll}
1 & 0 & 0
\end{array}\right], \boldsymbol{P}=\left[\begin{array}{ll}
0.5 & 0.5 \\
0.5 & 0.5
\end{array}\right], \\
\Sigma=\left[\begin{array}{ccc}
0 & 0 & 0 \\
0 & 0 & 0 \\
0 & 0.4 & 0.1
\end{array}\right] e \Psi=\left[\begin{array}{ccc}
0.3 & 0 & 0 \\
0 & 0.2 & 0 \\
0 & 0 & 0.3
\end{array}\right] .
\end{gathered}
$$

Neste caso, observamos que $(A, E, \mathbf{P})$ não é w-controlável e tomamos $(A, C, \mathbf{P})$ sdetetável, acarretando instabilidade do FK, a Figura (5.2.a) ilustra a limitação de $\mathcal{E}\{P(k)\}$, enquanto na Figura (5.2.b) verifica-se que $\mathcal{E}\{W(k, B, \psi)\}$ tende a crescer indefinidamente. 

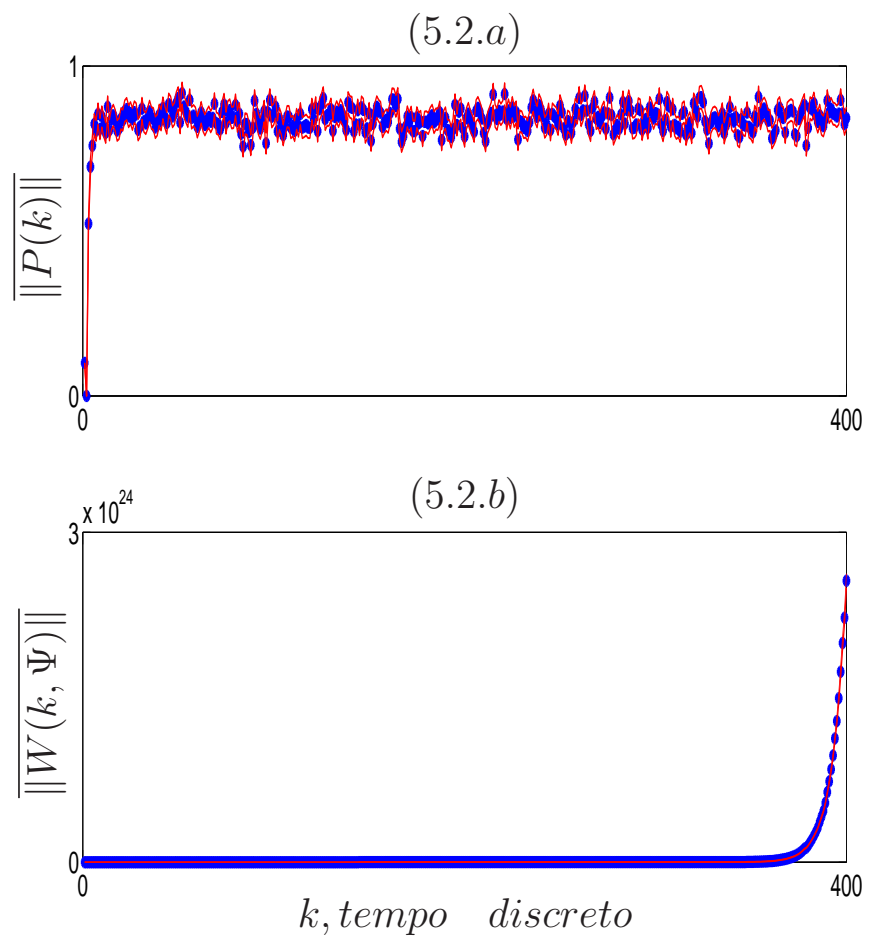

Figura 5.2: Exemplo 5.2: não w-controlável e s-detetável. Estimativas para $P(k)$ e $\mathcal{E}\{W(k, B, \Psi)\}$ e os respectivos desvios padrões marcados com linha contínua.

Exemplo 5.3. (Não w-controlável e s-detetável) Considere o sistema $\Phi_{M}$, como no Exemplo 5.1, com

$$
\begin{aligned}
& A_{1}=A_{2}=\left[\begin{array}{ccc}
0.9 & 0 & 0 \\
0 & 0 & 0 \\
0 & 0 & 0
\end{array}\right], E_{1}=\left[\begin{array}{ccc}
0 & 0 & 0 \\
0 & 0.1 & 0 \\
0 & 0 & 0
\end{array}\right], E_{2}=0, \\
& B_{1}=\left[\begin{array}{ccc}
0.3 & 0 & 0 \\
0 & 0.7 & 0 \\
0 & 0 & 0.7
\end{array}\right], B_{2}=\left[\begin{array}{ccc}
0.2 & 0 & 0 \\
0 & 0.8 & 0 \\
0 & 0 & 0.6
\end{array}\right], C_{1}=C_{2}=\left[\begin{array}{lll}
1 & 0 & 0
\end{array}\right], \\
& \boldsymbol{P}=\left[\begin{array}{ll}
0.4 & 0.6 \\
0.5 & 0.5
\end{array}\right], \Sigma=\left[\begin{array}{ccc}
0.2 & 0.1 & 0 \\
0.1 & 0 & 0 \\
0 & 0 & 0
\end{array}\right] \text { e } \Psi=\left[\begin{array}{ccc}
0.5 & 0 & 0 \\
0 & 0.8 & 0 \\
0 & 0 & 0.6
\end{array}\right]
\end{aligned}
$$

Neste caso, obtemos $(A, E, \mathbf{P})$ não w-controlável sob hipótese de s-detetabilidade e estabilidade do FK. Observe que este fato mostra que a necessidade das hipóteses não é satisfeita, a Figura (5.3.a) ilustra a limitação de $\mathcal{E}\{P(k)\}$, enquanto na Figura (5.3.b) tem-se a limitação de $\mathcal{E}\{W(k)\}$, confirmando a estabilidade do FK. 
$(5.3 . a)$

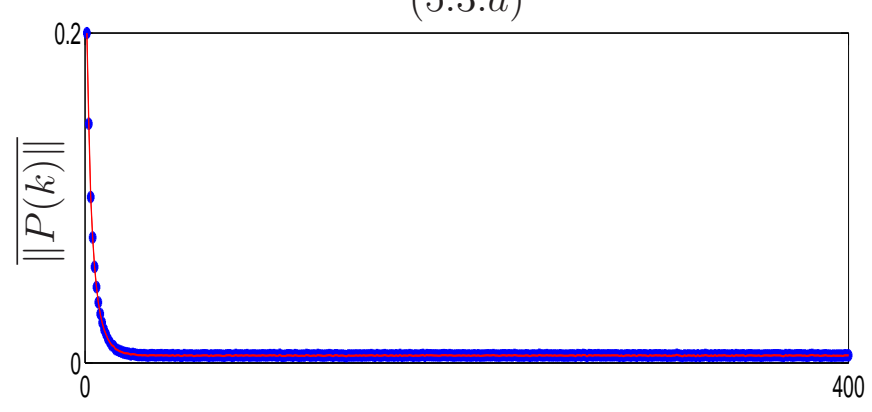

$(5.3 . b)$

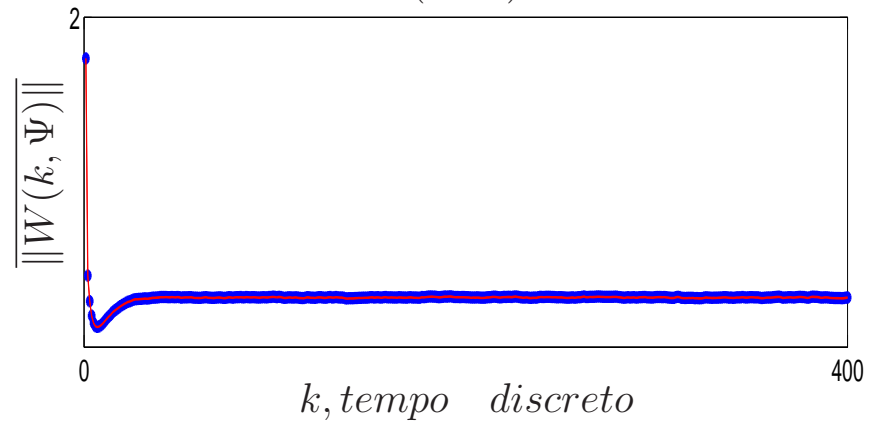

Figura 5.3: Exemplo 5.3: não w-controlável e s-detetável. Estimativas para $P(k)$ e $\mathcal{E}\{W(k, B, \Psi)\}$ e os respectivos desvios padrões marcados com linha contínua.

Exemplo 5.4. (W-controlável e não s-detetável) Considere o sistema $\Phi_{M}$, como no Exemplo 5.1, com

$$
\begin{gathered}
A_{1}=\left[\begin{array}{ccc}
0.2 & 0 & 0.2 \\
0 & 1.1 & 0 \\
0.1 & 0 & 0.9
\end{array}\right], A_{2}=\left[\begin{array}{ccc}
1.5 & 0 & 0 \\
0.1 & 0.9 & 0 \\
0.1 & 0 & 1.5
\end{array}\right], E_{1}=\left[\begin{array}{lll}
1 & 0 & 0 \\
1 & 1 & 0 \\
0 & 0 & 0.1
\end{array}\right], \\
E_{2}=\left[\begin{array}{ccc}
1 & 0 & 0 \\
0 & 1 & 0 \\
1 & 0 & 0.1
\end{array}\right], B_{1}=\left[\begin{array}{ccc}
1.2 & 0 & 0 \\
0 & 0.6 & 0 \\
0 & 0 & 1
\end{array}\right], B_{2}=\left[\begin{array}{ccc}
1.4 & 0 & 0 \\
0 & 0.7 & 0 \\
0 & 0 & 1.2
\end{array}\right], \\
C_{1}=C_{2}=\left[\begin{array}{ccc}
1 & 0 & 0
\end{array}\right], \boldsymbol{P}=\left[\begin{array}{cc}
0.3 & 0.7 \\
0.9 & 0.1
\end{array}\right], \\
\Sigma=\left[\begin{array}{ccc}
0 & 0 & 0.5 \\
0 & 0.6 & 0 \\
0 & 0.7 & 0.9
\end{array}\right] \quad \Psi=\left[\begin{array}{ccc}
0.9 & 0 & 0 \\
0 & 0.9 & 0 \\
0 & 0 & 0.9
\end{array}\right] .
\end{gathered}
$$

Este exemplo mostra que w-controlabilidade não é suficiente para estabilidade do FK, sem a hipótese de s-detetabilidade, a Figura (5.4.a) ilustra que $\mathcal{E}\{P(k)\}$ cresce indefinidamente e na Figura (5.4.b) observamos a divergência de $\mathcal{E}\{W(k, B, \Psi)\}$, concluindo na estabilidade do FK. 

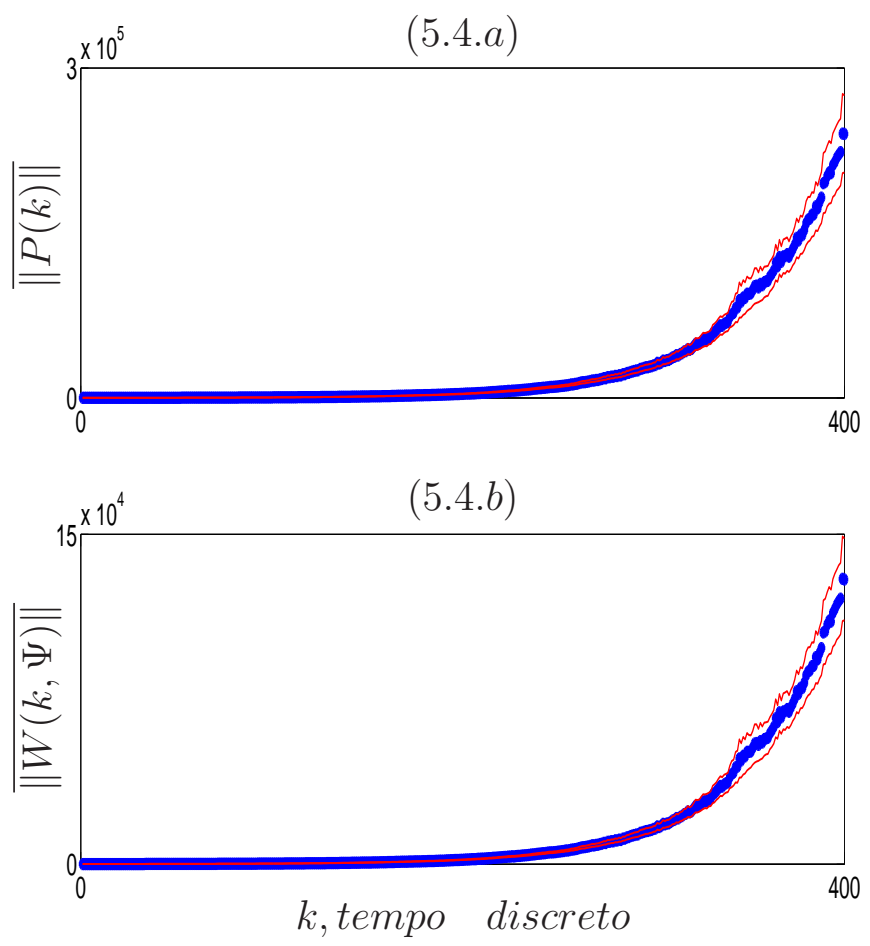

Figura 5.4: Exemplo 5.4: w-controlável e não s-detetável. Estimativas para $P(k)$ e $\mathcal{E}\{W(k, B, \Psi)\}$ e os respectivos desvios padrões marcados com linha contínua.

Resumidamente os exemplos apresentados ilustram a validade do resultado obtido no Teorema 2 w-controlabilidade e s-detetabilidade são condições suficientes para estabilidade do FK no sentido usual, sendo que o Exemplo 5.3 mostra que as condições não são necessárias. Na Tabela 5.2 sumariamos os resultados obtidos nos exemplos. ${ }^{1}$

Tabela 5.1: Tabela resumo: Estabilidade do FK

\begin{tabular}{|c|c|c|c|}
\hline Caso & w-controlabilidade & s-detetabilidade & estabilidade do FK \\
\hline \hline Exemplo 5.1 & $\checkmark$ & $\checkmark$ & $\checkmark$ \\
\hline Exemplo 5.2 & & $\checkmark$ & \\
\hline Exemplo 5.3 & & $\checkmark$ & $\checkmark$ \\
\hline Exemplo 5.4 & $\checkmark$ & & \\
\hline
\end{tabular}

\footnotetext{
"Obs: $\checkmark$ indica "sim".
} 


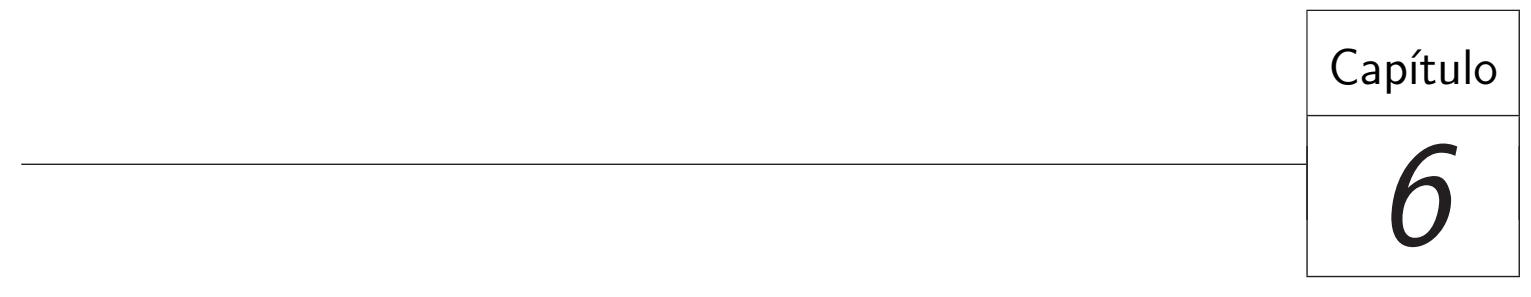

Conclusões

Resultados de estabilidade de filtros para sistemas lineares com saltos Markovianos a tempo discreto e espaço de estado finito formam uma questão de grande interesse, dadas as inúmeras aplicações. A estabilidade do FK para SLSM segue os mesmos padrões de estabilidade para SLD, no entanto, neste caso, a construção de um limitante para a covariância do erro de estimação $W(k)$ é abordada em função do valor esperado, uma vez que em função dos saltos de Markov, $W(k)$ forma um processo estocástico. Neste cenário buscamos condições suficientes para que exista um limitante superior para $W(k)$.

Exploramos as propriedades do conceito de w-controlabilidade, de forma a obter uma definição de w-controlabilidade viável para a construção dos principais resultados que envolvem estabilidade. Mostramos que w-controlabilidade é condição suficiente para positividade da covariância do erro de estimação $W(k)$ em instantes de tempo da forma $k=l n^{2} N$. Este resultado é fundamental para obtenção da trajetória auxiliar, que possibilita a caracterização de limitantes nos resultados de estabilidade.

Análises de casos sugerem que perturbações na condição inicial ou nos termos que acrescentam ruído ao sistema podem levar a instabilidade do FK, tornando-se essencial a obtenção de condições suficientes ou necessárias que garantam estabilidade. Assumimos a limitação do valor esperado da covariância de erro calculado $\mathcal{E}\{P(k)\}$ e w-controlabilidade de $(A, E, \mathbf{P})$ e através de trajetória auxiliar proveniente de um sistema w-controlável obtemos limitação para o valor esperado da covariância do erro de estimação real $W(k)$, para qualquer valor real de $\Psi$, o que fornece estabilidade com respeito a $\Psi$. Estes resultados implicam que w-controlabilidade e s-detetabilidade são em 
conjunto condições suficientes para estabilidade do FK. S-detetabilidade é claramente necessária, mas w-controlabilidade isoladamente não é necessária, veja Exemplo 4.5.

Consideramos também a estabilidade do FK no sentido mais usual (que contempla perturbações em $E$ ), para a qual apresentamos resultados parciais. De fato, apresentamos uma prova de que s-detetabilidade e w-controlabilidade garantam a estabilidade do FK, contudo assumindo uma hipótese sobre a cadeia e também uma conjectura.

Permanece em aberto a obtenção e demonstração de condições que sejam necessárias e suficientes para estabilidade do FK para sistemas lineares com saltos Markovianos para cadeias mais gerais e \ou sem a adição da hipótese na Conjectura 1. 


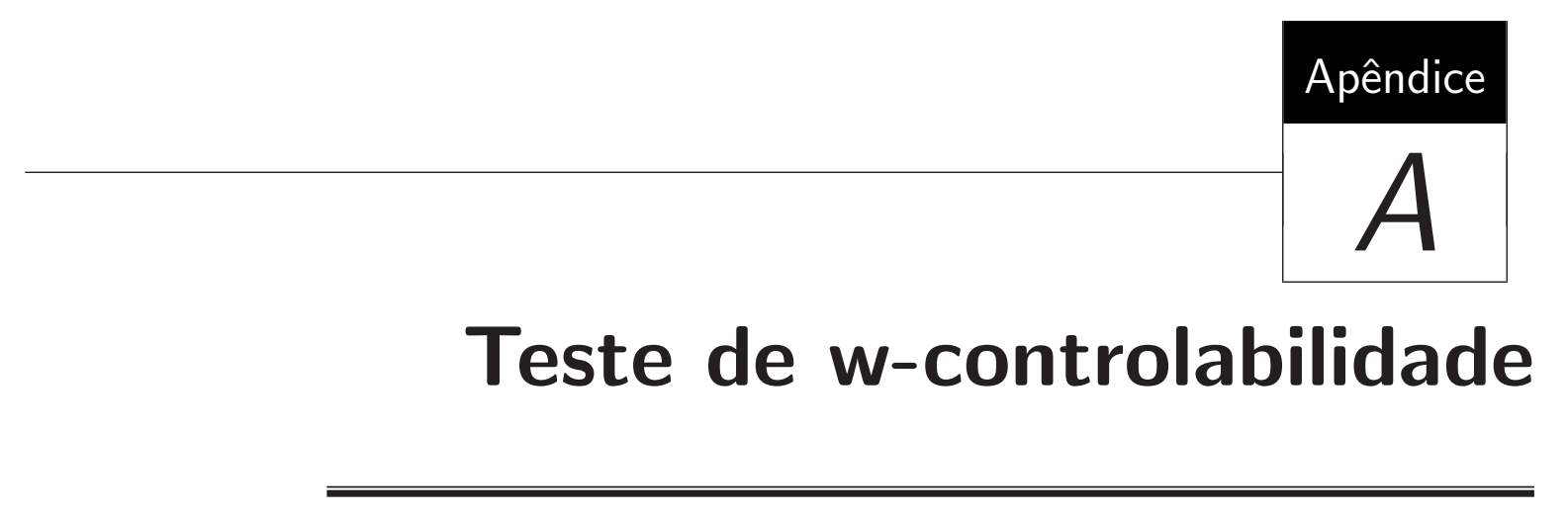

O algorítimo para verificação de w-controlabilidade apresentado abaixo, obedece as ideias do Lema 3.1 e foi utilizado para obtenção dos resultados nos exemplos.

\section{Método}

1. Considera-se o operador

$$
\begin{aligned}
S_{i}(k+1) & =\mathcal{T}_{A, i}(S(k))+\mathcal{T}_{E, i}(\mathbb{I}) \\
& =\sum_{j=1}^{N} p_{j i} A_{j} S_{i}(k) A_{j}^{\prime}+\sum_{j=1}^{N} E_{j} E_{j}^{\prime}, \quad i=1 \ldots N
\end{aligned}
$$

2. Pelo Lema 3.1, obtemos

(a) Se posto $S_{i}\left(n^{2} N\right)=n$ então (A,E,P) é w-controlável;

(b) Se $\operatorname{posto} S_{i}\left(n^{2} N\right)<n$ então $(\mathrm{A}, \mathrm{E}, \mathrm{P})$ não é w-controlável; 



\section{Referências Bibliográficas}

[1] B. D. O. Anderson and J. B. Moore. Optimal Filtering. Prentice-Hall, London, first edition, 1979.

[2] B. D. O. Anderson and J. B. Moore. Detectability and stabilizability of discretetime linear systems. SIAM Journal of Control and Optimization, 19(1):20-32, 1981.

[3] Castanon D. Dunn K. P. Greene C. S. Lee W. H. Sandell N. R. Athans, M. and A. S. Willsky. The stochastic control of the F-8C aircraft using a multiple model adaptive control (MMAC) method - Part i :Equilibrium flight. IEEE Transactions on Automatic Control, (22):798-780, 1977.

[4] C. T. Chen. Linear System Theory and Design. Oxford University Press, 1999.

[5] E. Cinlar. Introduction to Stochastic Processes. Prentice-Hall, 1975.

[6] E. F. Costa and A. Astolfi. A necessary and sufficient condition for semi-stability of the recursive Kalman filter. In Proc. ACC'08 American Control Conference, volume 11, pages 1280-1285, 2008.

[7] E. F. Costa and Alessandro Astolfi. On the stability of the recursive Kalman filter for linear time-invariant systems. In Proc. ACC'08 American Control Conference, volume 11, pages 1286 - 1291, 2008.

[8] E. F Costa and J. B. R do Val. Weak detectability and the linear quadratic control problem of discrete time Markov jump linear systems. International journal of control, page 1282, 2002.

[9] E. F. Costa, A. L. P. Manfrim, and J. B. R. do Val. Weak controllability and weak stabilizability concepts for linear systems with Markov jump parameters. In Proc. ACC'06 American Control Conference, Minneapolis,USA, 2006. 
[10] E.F Costa and A Astolfi. A bound for the error covariance of the recursive Kalman filter with Markov jump parameters. IEEE Transactions on Automatic Control, pages $37-42,2008$.

[11] E.F. Costa and A Astolfi. Characterization of exponential divergence of the Kalman filter for time-varying systems. SIAM Journal on Control and Optimazation, 48(5):2917-2944, 2009.

[12] O. L. V. Costa. Discrete-times coupled Riccati equations for systems with Markovia switching parameters. Journal of Mathematical Analysis and Applications, (149):197-216, 1983.

[13] O. L. V. Costa. Linear minimum mean square error estimation for discretetime Markovian jump linear systems. IEEE Transactions on Automatic Control, 39(8):1685-1689, 1994.

[14] O. L. V. Costa. Stability and control for linear systems with jump Markov pertubations. Stochastic Analysis and Applications, 1(13):91-110, 1995.

[15] O. L. V. Costa, M. D. Fragoso, and R. P. Marques. Discrete-Time Markovian Jump Linear Systems. Springer-Verlag, New York, 2005.

[16] O. L. V. Costa and M.D. Fragoso. Discrete-time LQ-optimal control problems for infinite Markov jump parameter systems. IEEE Transactions on Automatic Control, 40:2076-2088, 1995.

[17] O. L. V. Costa and R.P. Marques. Robust $H_{2}$-control for discrete-time Markovian jump linear systems. International Journal of Control, 73(1):11-21, 2000.

[18] O. L.V. Costa and M. D. Fragoso. Stability results for discrete-time linear systems with Markovian jumping parameters. Journal of Mathematical Analysis and Applications, 179:154-178, 1993.

[19] O.L.V. Costa and E.F. Tuesta. $H_{2}$-control and the separation principle for discrete time Markovian jump linear systems. Mathematics of Control, Signal and systems, (16):320-350, 2004.

[20] S. Costa, O.L.V. Guerra. Stationary filter for linear minimum mean square error estimator of discrete-time Markovian jump systems. Automatic Control, IEEE Transactions on, 47(8):1351-1356, 2002.

[21] C. E. de Souza. On stabilizing properties of solutions of the Riccati diference equation. IEEE Transactions on Automatic Control, 34(12):1313-1316, 1989. 
[22] J. do val and E. F. Costa. Numerical solutions for the linear-quadratic control problem of Markov jump linear systems and a weak detectability concept. IEEE Transactions on Automatic Control, 114(1):69-96, 2002.

[23] J. B. R do Val and T. Basar. Receding horizon control of jump linear systems and a macroeconomic policy problem. Journal of Economic Dynamics and Control, (23):1099-1131, 1999.

[24] J. B. R. do Val E. F. Costa and M. D. Fragoso. A new approach to detectability of discrete-time infinite Markov jump linear systems. SIAM Journal on Control and Optimization, Philadelphia, 43(6):2132-2156, 2005.

[25] R. J. Fitzgerald. Divergence of the Kalman filter. IEEE Transactions on Automatic Control, 16(6):736-747, 1971.

[26] A. C. Harvey. Forecasting, Structural Time Series Models and the Kalman Filter. Cambridge University Press, 1979.

[27] Chizeck H. J. Ji, Y. and K. A. Loparo. Stability and control of discrete-time jump linear systems. Control Theory and Advanced Technology, 2(7):247-270, 1991.

[28] Y. Ji, Feng X. Chizeck, H. J., and K. A. Loparo. Stability and control of discretetime jump lineat systems. Control Theory and Advanced tecnology, 2(7):247-296, 1983.

[29] Y. Ji and H. J. Chizeck. Controllability, observability and discrete- time Markovian jump linear quadratic control. International Journal of Control, 48(2):481-498, 1988.

[30] Y. Ji and H. J. Chizeck. Jump linear quadratic gaussian control: Steadystate solution and testable conditions. Control Theory and Advanced Technology, (6):289-319, 1990.

[31] P. R. Kumar and P. Varaiya. Stochastic Systems: Estimation, Identification, and Adaptive Control. Prentice-Hall, 1986.

[32] H. J. Kushner. Introduction to Stochastic Control. Holt,Rinehart and Winston, 1999.

[33] Neves M. Leonor, M. Construção de um pêndulo invertido sobre um robô móvel controlado com o executivo SHaRK. Revista do DETUA. 
[34] David G. Luenberger. Introduction to Dynamic Systems. Theory, Models, and Applications. 1979.

[35] S. Mastellone, P. Dorato, and C. T. Abdallah. Finite-time stochastic stability of discrete-time nonlinear systems: Analysis and design. 43th Conference on Decision and Control, 3:2572 - 2577, 2004.

[36] I. R. Petersen and A. V. Savkin. Robust Kalman Filtering for Signals and Systems with Large Uncertainties. Birkhauser, Boston, 1999.

[37] C. Price. An analysis of divergence problem in the Kalman filter. IEEE Transactions on Automatic Control, 13(6):699-702, 1968.

[38] I. R. Petersen R. Bitmead, M. R. Gevers and R. J. Kaye. Monotonicity and stabilizability properties of solutions of the riccati diference equation: Propositions, lemmas, theoremas, fallacious conjectures and counterexamples. Systems and Control Letters, 5:309-315, 1985.

[39] Arulamparam S. Ristic, B. and N Gordon. Beyond the Kalman Filter. Artech House Boston, 2004.

[40] S. Sangsuk-Iam and T. E. Bullock. Analysis of discrete-time Kalman filtering under incorrect noise covariances. IEEE Transactions on Automatic Control, 35(12):1304-1309, 1990.

[41] G.N Saridis. Intelligent robotic control. IEEE Transactions on Automatic Control, (28):547-557, 1983.

[42] A. H. Sayed. A framework for state-space estimation with uncertain models. IEEE Transactions on Automatic Control, 46(7):998-1013, 2001.

[43] D.D Sworder and R. O Rogers. An LQ-solution to a control problem associated with a solar thermal center receiver. IEEE Transactions on Automatic Control, 10(28):971-978, 1983.

[44] F. Wang and V. Balakrishnan. Robust Kalman filters for linear time-varying systems with stochastic parametric uncertainties. IEEE Transactions on Signal Processing, 50(4):803-813, 2002.

[45] J. L. Willems and F. M. Callier. Divergence of the stationary Kalman filter for correct and for incorrect noise variances. IMA Journal of Mathematical Control and Information, 1(9):47-54, 1992. 
[46] A.H. Zawiski. Stochastic Processes and Filtering Theory. Academic Press, 1970. 